The Astrophysical Journal, 652:1674-1693, 2006 December 1

(C) 2006. The American Astronomical Society. All rights reserved. Printed in U.S.A.

\title{
NEW DEBRIS DISKS AROUND NEARBY MAIN-SEQUENCE STARS: IMPACT ON THE DIRECT DETECTION OF PLANETS
}

\author{
C. A. Beichman, ${ }^{1}$ G. Bryden, ${ }^{2}$ K. R. Stapelfeldt ${ }^{2}$ T. N. Gautier ${ }^{2}$ K. Grogan, ${ }^{2}$ M. Shao, ${ }^{2}$ T. Velusamy, ${ }^{2}$ \\ S. M. Lawler, ${ }^{1}$ M. Blaylock, ${ }^{3}$ G. H. Rieke, ${ }^{3}$ J. I. Lunine, ${ }^{3}$ D. A. Fischer, ${ }^{4}$ G. W. Marcy, ${ }^{5}$ \\ J. S. Greaves, ${ }^{6}$ M. C. Wyatt, ${ }^{7}$ W. S. Holland, ${ }^{8}$ and W. R. F. Dent ${ }^{8}$ \\ Received 2006 April 17; accepted 2006 July 28
}

\begin{abstract}
Using the MIPS instrument on Spitzer, we have searched for infrared excesses around a sample of 82 stars, mostly F, G, and K main-sequence field stars, along with a small number of nearby M stars. These stars were selected for their suitability for future observations by a variety of planet-finding techniques. These observations provide information on the asteroidal and cometary material orbiting these stars, data that can be correlated with any planets that may eventually be found. We have found significant excess $70 \mu \mathrm{m}$ emission toward 12 stars. Combined with an earlier study, we find an overall $70 \mu \mathrm{m}$ excess detection rate of $13 \% \pm 3 \%$ for mature cool stars. Unlike the trend for planets to be found preferentially toward stars with high metallicity, the incidence of debris disks is uncorrelated with metallicity. By newly identifying four of these stars as having weak $24 \mu \mathrm{m}$ excesses (fluxes $\sim 10 \%$ above the stellar photosphere), we confirm a trend found in earlier studies wherein a weak $24 \mu \mathrm{m}$ excess is associated with a strong $70 \mu \mathrm{m}$ excess. Interestingly, we find no evidence for debris disks around 23 stars cooler than $\mathrm{K} 1$, a result that is bolstered by a lack of excess around any of the $38 \mathrm{~K} 1-\mathrm{M} 6$ stars in two companion surveys. One motivation for this study is the fact that strong zodiacal emission can make it hard or impossible to detect planets directly with future observatories such as the Terrestrial Planet Finder $(T P F)$. The observations reported here exclude a few stars with very high levels of emission, $>1000$ times the emission of our zodiacal cloud, from direct planet searches. For the remainder of the sample, we set relatively high limits on dust emission from asteroid belt counterparts.
\end{abstract}

Subject headings: circumstellar matter — infrared: stars — Kuiper Belt

\section{INTRODUCTION}

A planetary system is characterized by the properties of its parent star, by the number and nature of its gas giant and rocky planets, by the extent of its Kuiper and asteroid belts, and by the populations of gas and dust orbiting the central star. In the coming decade, astronomers will use a variety of techniques to address all of these aspects of neighboring solar systems. Initial results for gas giant planets are based on ground-based radial velocity searches. Eventually, nearby stars will be the targets of indirect and ultimately direct searches for terrestrial planets with the Space Interferometer Mission PlanetQuest (SIM PlanetQuest) and the Terrestrial Planet Finder (TPF). The Spitzer telescope is uniquely positioned to characterize the evolution, amount, structure, and composition of the dust associated with Kuiper and asteroid belts around many types of stars, including those with and without the gas giant planets now being detected by the radial velocity technique. Guaranteed Time Observer (GTO) studies such as the FGK sample (Beichman et al. 2005a; Bryden et al. 2006) and the

\footnotetext{
1 Michelson Science Center, California Institute of Technology, 770 South Wilson Avenue, Pasadena, CA 91125.

2 Jet Propulsion Laboratory, 4800 Oak Grove Drive, Pasadena, CA 91109.

3 Steward Observatory, University of Arizona, 933 North Cherry Avenue, Tucson, AZ 85721.

4 Department of Physics and Astronomy, San Francisco State University, San Francisco, CA 94132.

5 Department of Astronomy, University of California, Berkeley, CA 94720

6 School of Physics and Astronomy, University of St. Andrews, North Haugh, St. Andrews KY16 9SS, UK. $\mathrm{UK}$

${ }^{7}$ Institute of Astronomy, University of Cambridge, Cambridge CB3 0HA,

${ }^{8}$ UK Astronomy Technology Centre, Royal Observatory, Edinburgh EH9 $3 \mathrm{HJ}, \mathrm{UK}$
}

Nearby Stars program (T. N. Gautier et al. 2006, in preparation), plus the FEPS Legacy project (Meyer et al. 2004; Kim et al. 2005), have conducted photometric surveys of about 200 nearby stars at 24 and $70 \mu \mathrm{m}$. The photometric survey discussed here uses Spitzer images at 24 and $70 \mu \mathrm{m}$ to look for debris disks around an additional 82 stars, rounding out existing surveys of the closest stars.

As the Spitzer programs are completed, we will be able to carry out statistical investigations of the debris disk phenomenon in terms of the age, metallicity, and spectral type of parent stars. In particular, by nearly doubling the size of the existing sample of stars (relative to the ongoing GTO/Legacy programs), we can hope to identify and improve the statistics of types of excess that appear to be rare based on existing Infrared Astronomical Satellite (IRAS) or Infrared Space Observatory (ISO) observations, e.g., hot dust, extremely large disk-to-star luminosity ratios $\left(L_{\text {dust }} / L_{*}\right)$ around mature stars (Fajardo-Acosta et al. 2000; Habing et al. 2001; Spangler et al. 2001), or low-mass stars. The incidence of excesses at the IRAS/ISO sensitivity level is about 15\% (Backman \& Paresce 1993; Bryden et al. 2006) so that a total Spitzer sample of 250-300 stars can hope to identify over 50 stars with excesses suitable for future study. Stars with hot excesses ( peak wavelength $<24 \mu \mathrm{m}$ ) are considerably rarer, 2\%-3\% (Fajardo-Acosta et al. 2000; Laureijs et al. 2002; Beichman et al. 2006), so that a survey of a large number of stars is needed to generate a statistically meaningful sample.

As the statistics of planets build up, we will be able to correlate the properties of debris disks (total mass, physical configuration, composition) with properties of number, location, and mass of planets. Much lower dust masses can be detected with Spitzer than was previously possible, particularly for solar-type and cooler stars. Beichman et al. (2005a) and Bryden et al. (2006) have shown 
that with Spitzer instruments, we can reach just a few times the fractional luminosity predicted for our own Kuiper Belt $[(0.3-5) \times$ $10^{-6}$; Backman \& Paresce 1993; Stern 1996]. Determining how many mature stars like the Sun have Kuiper Belts comparable to our own is an important ingredient in understanding the formation and evolution of solar systems like our own (Levison \& Morbidelli 2003).

Finally, the Spitzer data will help us to understand the potential influence of zodiacal emission on the eventual direct detectability of planets. As highlighted in a number of $T P F$ studies, including the "Precursor Science Roadmap for TPF" (Lawson et al. 2004), ${ }^{9}$ the level of exozodiacal emission can affect the ability of TPF to detect planets directly, particularly for extreme cases with much greater dust contamination than in the solar system. A complete census of potential TPF stars will assist in the eventual selection of TPF targets by determining or setting a limit to the amount of exozodiacal emission around each star.

This paper focuses on the results of the 24 and $70 \mu \mathrm{m}$ survey using the Multiband Imaging Photometer for Spitzer (MIPS; Rieke et al. 2004). After describing our target selection ( $\S 2$ ), we present these MIPS observations in $\S 3$. As is discussed below, a number of sources in this sample appear to be extended. These are highlighted in $\S 3$ but are discussed in detail in a separate paper (G. Bryden et al. 2006, in preparation). Follow-up observations of sources with excesses using the IRS spectrometer are just now being completed; these will also be detailed in a later paper. In $\S 4$ we discuss how our MIPS flux measurements constrain the dust properties in each system. For the systems identified here as having IR excess, combined with those from Bryden et al. (2006), $\S 5$ attempts to find correlations between the dust emission and system parameters such as stellar metallicity, spectral type, and age. Finally, in $\S 6$ we assess the influence of debris disks on the detectability of planets.

\section{STELLAR SAMPLE}

Our sample is based on work carried out by radial velocity search teams (e.g., Marcy et al. 2004) and by the SIM PlanetQuest and TPF Science Teams to identify the most suitable targets for the indirect or direct detection of terrestrial mass planets $\left(1-10 M_{\oplus}\right)$. One target list consists of the 100 stars in the SIM PlanetQuest Tier-1 sample, which will be the most intensively observed stars in the two SIM PlanetQuest projects dedicated to finding planets around nearby stars (Marcy et al. 2002; Shao et al. 2002). ${ }^{10}$ Since the absolute astrometric signal from a planet scales as $3 \mu \mathrm{s}\left(D_{*} / \mathrm{pc}\right)^{-1}\left(a_{\text {planet }} / \mathrm{AU}\right)\left(M_{\text {planet }} / M_{\oplus}\right)\left(M_{\odot} / M_{*}\right)$, the SIM PlanetQuest teams are concentrating on some of the closest, lower mass stars for their deepest surveys for terrestrial planets. Thus, the SIM PlanetQuest list includes a number of late $\mathrm{K}$ and $\mathrm{M}$ stars not included in the other Spitzer samples or in TPF lists.

We also draw from a number of lists prepared by Science Working Groups for the TPF-Coronagraph $(T P F-C)$ and $T P F$ Interferometer (TPF-I) missions. Although the TPF lists are not definitive given the indeterminate status of the project, the outline of the sample is clear (Beichman et al. 2005c; Traub et al. 2006). We start with F0-M5 stars of luminosity classes IV or V and refine the list by making a few simple assumptions about the nature of planetary systems and the properties of TPF. Specifically, we (1) exclude stars with binary companions within $100 \mathrm{AU}$ as being inimical to the formation or stable evolution of planetary

\footnotetext{
9 Available at http://planetquest.jpl.nasa.gov/documents/RdMp273.pdf.

10 The merged, high-priority target list for these projects is available at http:// astron.berkeley.edu/ gmarcy/sim_draft.html.
}

systems, (2) require that the angular extent of the habitable zone (Kasting et al. 1993; $\sim 1$ AU for a $1 L_{\odot}$ luminosity star, scaled by the square root of the stellar luminosity) exceed 50 mas, and (3) impose an outer distance cutoff of $25 \mathrm{pc}$ (although we allowed a few F0-F5 stars at distances as great as $30 \mathrm{pc}$ to bring up their numbers). To enable good measurements with Spitzer, we rejected stars with high levels of stellar and/or cirrus confusion based on examination of IRAS maps.

Comparison of potential SIM PlanetQuest and TPF targets in cirrus-free sky with the Spitzer Reserved Object Catalog (as of 2003 November) showed 81 stars with spectral types ranging from F0 to M3.5, as listed in Table 1. One more star, GL 436, was added through a Director's Discretionary Time proposal after the discovery of a planet in this system was announced (Butler et al. 2004). Binary companions within the 82 fields of view have also been included as secondary targets; six such companions are identified as bright enough for clear detection in both the 24 and $70 \mu$ m images. ${ }^{11}$ The divergent proper motion of HD 48682B, an M0 star 30" to the northeast of HD 48682, rules out a physical association between the two stars. Thus, HD 48682B is not included in this sample. The true binarity of the other six neighboring sources is verified via their Hipparcos distances and space-motion measurements. Angular separations in these systems range from $10^{\prime \prime}$ to $100^{\prime \prime}$, with projected orbital separations between 100 and 1000 AU. Data for the companions are listed separately at the end of Tables 1 and 2. With their inclusion, our total sample contains 88 stars within 82 targeted fields.

Binned by spectral type, the SIM PlanetQuest/TPF sample consists of $37 \mathrm{~F}$ stars, $19 \mathrm{G}$ stars, $24 \mathrm{~K}$ stars, and eight M stars. Typical distances range from 10 to $20 \mathrm{pc}$, closer for $\mathrm{M}$ and $\mathrm{K}$ stars and farther for earlier spectral types. Figure 1 shows the overall distribution of observed spectral types. Some basic parameters of the sample stars are listed in Table 1, most importantly age and metallicity, which are also shown as histograms in Figures 2 and 3. There is no explicit target selection based on stellar age or metallicity, but known planet-bearing stars have been specifically included in a couple of cases. Of this sample, only two stars (GJ 436 and HD 147513) are already known to have planets; most of the other stars with planets either are too faint, lie in cirruscontaminated regions, or are already observed in other Spitzer programs (e.g., Beichman et al. 2005a).

In this paper we first discuss the 88 primary and secondary stars and then add in the stars observed in Bryden et al. (2006) to increase the size of the sample for some of the statistical discussions.

\section{SPITZER OBSERVATIONS}

All stars were observed with MIPS at $24 \mu \mathrm{m}$ and, with one exception (HD 265866), at $70 \mu \mathrm{m}$. In order to help pin down their stellar photospheres, four M stars, GJ 908, HD 36395, HD 191849 , and HD 265866, were also observed with the Infrared Array Camera (IRAC) in subarray mode at all four of its wavelengths $(3.6,4.5,5.8$, and $8.0 \mu \mathrm{m})$. Seven stars identified as having IR excess were observed with IRS, the Spitzer spectrograph, as follow-up observations, as detailed in a future paper.

\footnotetext{
11 The $24 \mu \mathrm{m}$ image of HD 265866 has what appears to be an equal-brightness binary companion located $40^{\prime \prime}$ northwest of the target primary star. However, there is no visible or near-IR neighboring source. In fact, the second $24 \mu \mathrm{m}$ source is a chance alignment with a passing asteroid. Software specifically developed for locating asteroids relative to the Spitzer observatory (part of the Horizons package; Giorgini 2005; available at http://ssd.jpl.nasa.gov/ispy.html) identifies this object as asteroid 11847 ("Winckelmann:" $H=13.4, a=2.67 \mathrm{AU}, e=0.065, i=10.23$; Vizier Online Data Catalog, B/astorb [E. Bowell 1996]).
} 
TABLE 1

Basic Data

\begin{tabular}{|c|c|c|c|c|c|c|c|c|c|c|c|c|c|c|c|}
\hline \multirow[b]{3}{*}{ STAR } & \multirow[b]{3}{*}{ HIP } & \multirow[b]{3}{*}{ GJ } & \multirow[b]{3}{*}{ HR } & \multirow[b]{3}{*}{ OtHER NAME } & \multirow[b]{3}{*}{ SPeCtral Type } & \multirow{3}{*}{$\begin{array}{c}V \\
(\mathrm{mag})\end{array}$} & \multirow{3}{*}{$\begin{array}{c}K \\
(\mathrm{mag})\end{array}$} & \multicolumn{5}{|c|}{ Age } & \multirow{2}{*}{\multicolumn{2}{|c|}{$[\mathrm{Fe} / \mathrm{H}]$}} & \multirow[b]{3}{*}{ REFERENCES } \\
\hline & & & & & & & & \multirow{2}{*}{$\begin{array}{c}\mathrm{Mo} / \text { W/Average } \mathrm{e}^{\mathrm{a}} \\
\text { (Gyr) }\end{array}$} & \multirow{2}{*}{$\begin{array}{l}\text { Min } \\
(\text { Gyr })\end{array}$} & \multirow{2}{*}{$\begin{array}{l}\text { Max } \\
(\mathrm{Gyr})\end{array}$} & \multirow{2}{*}{$\begin{array}{l}\text { Number of } \\
\text { Estimates }\end{array}$} & \multirow[b]{2}{*}{ References } & & & \\
\hline & & & & & & & & & & & & & Average & $\sigma$ & \\
\hline GL $436^{\mathrm{b}} \ldots \ldots \ldots \ldots \ldots \ldots$ & 57087 & 436 & $\ldots$ & & M2.5 & 10.67 & 6.07 & $\ldots$ & $\ldots$ & $\ldots$ & 0 & & $\ldots$ & $\ldots$ & \\
\hline GL $908 \ldots \ldots \ldots \ldots \ldots \ldots$ & 117473 & 908 & $\ldots$ & BR Psc & M1 & 8.98 & 5.04 & & & & 0 & & & & \\
\hline HD $739 \ldots \ldots \ldots \ldots$ & 950 & 3013 & 35 & $\theta \mathrm{Scl}$ & $\mathrm{F} 4 \mathrm{~V}$ & 5.24 & $4.13^{\mathrm{c}}$ & 2.80 & 2.40 & 3.19 & 2 & 6,11 & -0.13 & 0.07 & $3,4,6,9,11,15$ \\
\hline 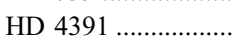 & 3583 & 1021 & 209 & & G5 IV & 5.80 & $4.30^{\mathrm{c}}$ & 12.30 & & & 1 & 11 & -0.17 & 0.07 & $4,11,14,15$ \\
\hline 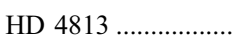 & 3909 & 37 & 235 & $19 \mathrm{Cet}$ & F7 IV-V & 5.17 & $4.02^{\mathrm{c}}$ & 5.04 & 2.35 & 9.63 & 7 & $2,6,7,8,11,12$ & -0.16 & 0.09 & $3,2,4,6,7,8,9,11,12,15$ \\
\hline HD $10360^{\mathrm{d}} \ldots \ldots \ldots \ldots \ldots$ & & $66 \mathrm{~B}$ & 486 & $\ldots$ & K2 V & 5.76 & $3.56^{\mathrm{c}}$ & 0.15 & $\ldots$ & $\ldots$ & 1 & 10 & -0.23 & 0.03 & $4,11,14,15,16$ \\
\hline HD $16895 \ldots \ldots \ldots \ldots \ldots$ & 12777 & $107 \mathrm{~A}$ & 799 & $\theta$ Per & F7 V & 4.10 & $2.98^{\mathrm{e}}$ & 5.01 & 2.50 & 7.94 & 5 & $17,2,7,11,12$ & -0.08 & 0.09 & $3,2,4,7,9,11,12,15,16$ \\
\hline HD $20794 \ldots \ldots \ldots \ldots . . . . .$. & 15510 & 139 & 1008 & e Eri & G8 V & 4.26 & $2.52^{\mathrm{e}}$ & & $\ldots$ & & 0 & & -0.32 & 0.09 & $3,4,6,11,12,14,15,16$ \\
\hline HD $22001 \ldots \ldots \ldots \ldots$ & & $143.2 \mathrm{~A}$ & 1083 & $\kappa$ Ret & F5 IV-V & 4.71 & $3.94^{\mathrm{c}}$ & 0.60 & 0.60 & 9.38 & 5 & $10,8,11$ & -0.13 & 0.07 & $3,8,9,11,15$ \\
\hline HD $23249 \ldots \ldots \ldots \ldots$ & 17378 & 150 & 1136 & $\delta$ Eri & K0 IV & 3.52 & $1.45^{\mathrm{e}}$ & 12.59 & $\ldots$ & $\ldots$ & 1 & 12 & 0.02 & 0.11 & $3,12,14,16$ \\
\hline HD $23754 \ldots \ldots \ldots \ldots \ldots$ & 17651 & 155 & 1173 & 27 Eri & F3/F5 V & 4.22 & $3.35^{\mathrm{c}}$ & 2.01 & 1.40 & 3.02 & 4 & $5,6,9,11$ & 0.05 & 0.08 & $3,5,6,9,11,15$ \\
\hline HD $25998 \ldots \ldots \ldots \ldots . . . .$. & 19335 & 161 & 1278 & 50 Per & F7 V & 5.52 & $4.28^{\mathrm{c}}$ & 0.60 & 0.60 & 5.14 & 4 & $10,2,7,11$ & -0.01 & 0.10 & $3,2,7,9,11,15$ \\
\hline HD $28343 \ldots \ldots \ldots \ldots$ & 20917 & 169 & $\ldots$ & $\ldots$ & K7 V & 8.30 & 4.88 & $\ldots$ & $\ldots$ & $\ldots$ & 0 & & $\ldots$ & $\ldots$ & \\
\hline HD $32147 \ldots \ldots \ldots \ldots \ldots$ & 23311 & 183 & 1614 & $\ldots$ & $\mathrm{K} 3 \mathrm{~V}$ & 6.22 & $3.71^{\mathrm{c}}$ & $\ldots$ & $\ldots$ & $\ldots$ & 0 & & 0.16 & 0.14 & $3,4,6,12,15,16$ \\
\hline HD $36395 \ldots \ldots \ldots \ldots . .$. & 25878 & 205 & $\ldots$ & $\ldots$ & $\mathrm{M} 1.5 \mathrm{~V}$ & 7.97 & $3.86^{\mathrm{e}}$ & $\ldots$ & $\ldots$ & $\ldots$ & 0 & & 0.60 & $\ldots$ & 3 \\
\hline HD $38392^{\mathrm{d}} \ldots \ldots \ldots \ldots \ldots$ & & $216 \mathrm{~B}$ & 1982 & $\gamma$ Lep B & $\mathrm{K} 2 \mathrm{~V}$ & 6.15 & $4.13^{\mathrm{c}}$ & 8.94 & 8.75 & 9.14 & 3 & 8 & -0.05 & 0.09 & $3,4,8,9,11,14,15$ \\
\hline HD $38858 \ldots \ldots \ldots \ldots$ & 27435 & 1085 & 2007 & $\ldots$ & G4 V & 5.97 & $4.41^{\mathrm{c}}$ & 4.57 & 3.19 & 12.20 & 3 & $17,6,11$ & -0.25 & 0.01 & $6,11,15,16$ \\
\hline 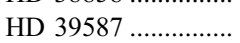 & 27913 & 222 & 2047 & 54 Ori & G0 V & 4.39 & $2.97^{\mathrm{e}}$ & 6.60 & 0.10 & 10.70 & 5 & $1,2,7,11,12$ & -0.07 & 0.07 & $3,2,4,7,9,11,12,15,16$ \\
\hline HD $40136 \ldots \ldots \ldots \ldots \ldots$ & 28103 & 225 & 2085 & $\eta$ Lep & F1 V & 3.71 & $2.90^{\mathrm{e}}$ & 1.31 & 1.22 & 1.41 & 3 & $6,11,12$ & -0.16 & 0.06 & $3,6,9,11,12$ \\
\hline 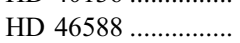 & 32439 & 240 & 2401 & & F8 V & 5.44 & $4.14^{\mathrm{c}}$ & 5.13 & 4.27 & 6.20 & 4 & $1,5,6,11$ & -0.22 & 0.07 & $5,6,9,11,14$ \\
\hline HD $48682 \ldots \ldots \ldots \ldots \ldots$ & 32480 & 245 & 2483 & 56 Aur & G0 V & 5.24 & $4.13^{\mathrm{c}}$ & 3.31 & 3.31 & 8.91 & 5 & $17,1,9,11,12$ & 0.07 & 0.08 & $3,4,9,11,12,15,16$ \\
\hline HD $50281^{\mathrm{d}}$. & 32984 & $250 \mathrm{~A}$ & $\ldots$ & $\ldots$ & K3 V & 6.58 & $4.11^{\mathrm{c}}$ & 9.42 & 9.02 & 9.82 & 3 & 8 & 0.06 & 0.07 & $3,8,9,15,16$ \\
\hline HD $53706^{\mathrm{d}} \ldots \ldots \ldots \ldots \ldots$ & 34069 & $264.1 \mathrm{~B}$ & 2668 & $\ldots$ & $\mathrm{K} 0 \mathrm{~V}$ & 6.83 & 4.94 & & & & 0 & & -0.24 & 0.05 & $3,4,11,14,15,16$ \\
\hline HD $55892 \ldots \ldots \ldots \ldots$. & 34834 & 268 & 2740 & QW Pup & F0 IV & 4.49 & $3.71^{\mathrm{c}, \mathrm{e}}$ & 1.78 & 1.40 & 2.16 & 2 & 7,11 & -0.30 & 0.10 & $3,7,9,11$ \\
\hline 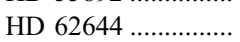 & 37606 & & 2998 & GJ 284 & G6 IV & 5.04 & $3.12^{\mathrm{c}}$ & 7.17 & 3.41 & 14.13 & 3 & $6,12,13$ & -0.09 & 0.21 & $3,6,12,13,15$ \\
\hline 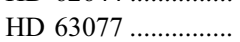 & 37853 & $288 \mathrm{~A}$ & 3018 & 171 Pup & G0 V & 5.36 & $3.75^{\mathrm{c}}$ & 5.01 & 5.01 & 14.50 & 4 & $17,2,6,11$ & -0.79 & 0.11 & $3,2,4,6,9,11,12,15$ \\
\hline HD $67228 \ldots \ldots \ldots$ & 39780 & & 3176 & $\mu \mathrm{Cnc}$ & G2 IV & 5.30 & $3.83^{\mathrm{e}}$ & 8.32 & 5.50 & 8.32 & 4 & $17,5,6,11$ & 0.11 & 0.06 & $3,5,6,11,15,16$ \\
\hline 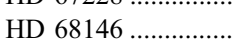 & 40035 & $297.2 \mathrm{~A}$ & 3202 & 18 Pup & F7 V & 5.53 & $4.35^{\mathrm{c}}$ & 4.18 & 2.92 & 5.19 & 4 & $2,7,9,11$ & -0.13 & 0.10 & $3,2,4,7,9,11,15$ \\
\hline 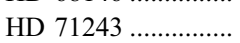 & 40702 & 305 & 3318 & $\alpha$ Cha & F5 V & 4.05 & $3.15^{\mathrm{c}}$ & 1.47 & 1.40 & 1.53 & 2 & 5,11 & 0.07 & 0.02 & $5,9,11$ \\
\hline HD $72673 \ldots \ldots \ldots \ldots$ & 41926 & 309 & 3384 & $\ldots$ & K0 V & 6.38 & $4.44^{\mathrm{c}}$ & 4.57 & $\ldots$ & & 1 & 17 & -0.36 & 0.06 & $3,4,6,11,14,15,16$ \\
\hline HD $76653 \ldots \ldots$ & 43797 & 3519 & 3570 & $\ldots$ & F6 V & 5.70 & $4.56^{\mathrm{c}}$ & 2.31 & 2.10 & 2.52 & 2 & 6,11 & -0.04 & 0.07 & $6,9,11$ \\
\hline 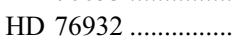 & 44075 & 3523 & 3578 & $\ldots$ & F7/F8 IV/V & 5.80 & $4.36^{\mathrm{c}}$ & 11.00 & 9.29 & 12.50 & 4 & $2,5,6,11$ & -0.84 & 0.12 & $3,2,4,5,6,7,9,11,15$ \\
\hline HD $78366 \ldots \ldots \ldots \ldots \ldots$ & 44897 & 334 & 3625 & $\ldots$ & F9 V & 5.95 & 4.55 & 5.17 & 3.84 & 6.50 & 2 & 6,11 & 0.02 & 0.09 & $4,6,9,11,16$ \\
\hline HD $79211^{\mathrm{d}} \ldots \ldots \ldots \ldots \ldots$ & 120005 & $338 \mathrm{~B}$ & $\ldots$ & $\ldots$ & K2 & 7.70 & $4.14^{\mathrm{e}}$ & & $\ldots$ & . & 0 & & $\ldots$ & $\ldots$ & \\
\hline 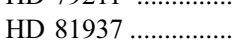 & 46733 & 3559 & 3757 & h UMa & F0 IV & 3.65 & $2.82^{\mathrm{c}, \mathrm{e}}$ & 0.90 & $\begin{array}{l}\cdots \\
\ldots\end{array}$ & $\cdots$ & 1 & 11 & 0.06 & $\begin{array}{l}\cdots \\
\ldots\end{array}$ & 11 \\
\hline 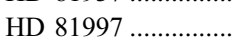 & 46509 & $348 \mathrm{~A}$ & 3759 & $31 \mathrm{Hya}$ & F6 V & 4.59 & $3.56^{\mathrm{c}}$ & 6.38 & 1.94 & 9.43 & 5 & $8,9,11$ & 0.00 & 0.01 & $4,8,9,11$ \\
\hline HD $85512 \ldots \ldots \ldots \ldots$ & 48331 & $\ldots$ & $\ldots$ & GJ 370 & $\mathrm{~K} 5 \mathrm{~V}$ & 7.67 & 4.72 & 0.30 & $\ldots$ & $\ldots$ & 1 & 10 & $\ldots$ & $\ldots$ & \\
\hline 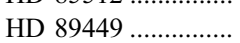 & 50564 & 388 & 4054 & 40 Leo & F6 IV & 4.78 & $3.65^{\mathrm{c}, \mathrm{e}}$ & 2.31 & 1.64 & 3.40 & 4 & $5,6,9,11$ & 0.02 & 0.08 & $3,5,6,9,11,15$ \\
\hline HD 90089 & 51502 & 392 & 4084 & $\ldots$ & $\mathrm{F} 2 \mathrm{~V}$ & 5.25 & $4.27^{\mathrm{c}}$ & 1.78 & 1.50 & 2.06 & 2 & 6,11 & -0.28 & 0.10 & $6,9,11,15$ \\
\hline
\end{tabular}


TABLE 1-Continued

\begin{tabular}{|c|c|c|c|c|c|c|c|c|c|c|c|c|c|c|c|}
\hline \multirow[b]{3}{*}{ Star } & \multirow[b]{3}{*}{ HIP } & \multirow[b]{3}{*}{ GJ } & \multirow[b]{3}{*}{ HR } & \multirow[b]{3}{*}{ Other NAME } & \multirow[b]{3}{*}{ Spectral Type } & \multirow{3}{*}{$\begin{array}{c}V \\
(\mathrm{mag})\end{array}$} & \multirow{3}{*}{$\begin{array}{c}K \\
(\mathrm{mag})\end{array}$} & \multicolumn{5}{|c|}{ AgE } & \multirow{2}{*}{\multicolumn{2}{|c|}{$[\mathrm{Fe} / \mathrm{H}]$}} & \multirow[b]{3}{*}{ REFERENCES } \\
\hline & & & & & & & & \multirow{2}{*}{$\begin{array}{c}\mathrm{Mo} / \mathrm{W} / \text { Average }^{\mathrm{a}} \\
\text { (Gyr) }\end{array}$} & \multirow{2}{*}{$\begin{array}{l}\text { Min } \\
(\mathrm{Gyr})\end{array}$} & \multirow{2}{*}{$\begin{array}{l}\text { Max } \\
\text { (Gyr) }\end{array}$} & \multirow{2}{*}{$\begin{array}{l}\text { Number of } \\
\text { Estimates }\end{array}$} & \multirow[b]{2}{*}{ References } & & & \\
\hline & & & & & & & & & & & & & Average & $\sigma$ & \\
\hline 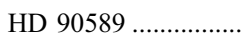 & 50954 & 391 & 4102 & I Car & F2 IV & 3.99 & $3.12^{\mathrm{e}}$ & 1.73 & 0.40 & 3.33 & 3 & $6,9,11$ & 0.01 & 0.14 & $6,9,11$ \\
\hline HD $91324 \ldots \ldots \ldots \ldots \ldots . .$. & 51523 & 397 & 4134 & $\ldots$ & F6 V & 4.89 & $3.58^{\mathrm{c}}$ & 5.39 & 4.28 & 7.94 & 4 & $7,9,11,12$ & -0.54 & 0.35 & $3,7,9,11,12,15$ \\
\hline 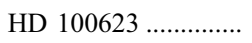 & 56452 & $432 \mathrm{~A}$ & 4458 & $\ldots$ & $\mathrm{K} 0 \mathrm{~V}$ & 5.96 & $4.02^{\mathrm{c}}$ & 3.72 & 3.72 & 10.08 & 4 & 17,8 & -0.38 & 0.10 & $4,8,9,11,14,15,16$ \\
\hline HD $102365 \ldots \ldots \ldots \ldots . . . .$. & 57443 & $442 \mathrm{~A}$ & 4523 & $\ldots$ & G5 V & 4.89 & $3.31^{\mathrm{e}}$ & 8.95 & 6.12 & 10.08 & 4 & 6,8 & -0.36 & 0.14 & $3,4,6,8,11,12,14,15,16$ \\
\hline HD $103095 \ldots \ldots \ldots \ldots . . .$. & 57939 & $451 \mathrm{~A}$ & 4550 & CF UMa & G8 V & 6.42 & $4.37^{\mathrm{e}}$ & 3.24 & 3.24 & 5.40 & 2 & 17,1 & -1.35 & 0.02 & $3,4,11,12,15,16$ \\
\hline HD $105211 \ldots \ldots \ldots \ldots \ldots$ & 59072 & 455 & 4616 & $\eta \mathrm{Cru}$ & $\mathrm{F} 2 \mathrm{~V}$ & 4.14 & $3.20^{\mathrm{c}}$ & 2.53 & 1.30 & 3.99 & 3 & $5,6,11$ & -0.37 & 0.18 & $6,9,11$ \\
\hline HD $105452 \ldots \ldots \ldots \ldots \ldots . .$. & 59199 & 455 & 4623 & $\alpha \operatorname{Crv}$ & F0 IV/V & 4.02 & $3.17^{\mathrm{e}}$ & 2.82 & & $\ldots$ & 1 & 12 & -0.43 & 0.26 & $3,11,12$ \\
\hline HD $109085 \ldots \ldots \ldots \ldots$ & 61174 & 471 & 4775 & $\eta \mathrm{Crv}$ & F2 V & 4.30 & $3.54^{\mathrm{e}}$ & 1.27 & 0.95 & 1.56 & 3 & $6,9,11$ & -0.05 & 0.04 & $6,9,11$ \\
\hline HD $129502 \ldots \ldots \ldots \ldots . .$. & 71957 & 9491 & 5487 & $\mu$ Vir & $\mathrm{F} 2 \mathrm{~V}$ & 3.87 & $2.90^{\mathrm{e}}$ & 1.29 & 0.71 & 1.71 & 4 & $5,6,9,11$ & 0.03 & 0.09 & $5,6,9,11$ \\
\hline HD $131977^{\mathrm{d}} \ldots$ & 73184 & $570 \mathrm{~A}$ & 5568 & $\ldots$ & $\mathrm{K} 4 \mathrm{~V}$ & 5.72 & $3.15^{\mathrm{e}}$ & $\ldots$ & $\ldots$ & $\ldots$ & 0 & & 0.07 & 0.07 & $3,12,14,15,16$ \\
\hline HD $132254 \ldots \ldots \ldots \ldots \ldots . . . .$. & 73100 & 3880 & 5581 & $\ldots$ & F7 V & 5.63 & 4.41 & 3.35 & 2.16 & 3.96 & 6 & $2,5,6,7,9,11$ & 0.01 & 0.05 & $3,2,5,7,9,11,14,15$ \\
\hline HD $136352 \ldots \ldots \ldots \ldots . . . .$. & 75181 & 582 & 5699 & $\ldots$ & $\mathrm{G} 2 \mathrm{~V}$ & 5.65 & $4.16^{\mathrm{c}}$ & 11.71 & 7.52 & 15.90 & 2 & 6,11 & -0.36 & 0.08 & $3,4,6,11,12,14,15,16$ \\
\hline 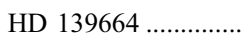 & 76829 & 594 & 5825 & g Lup & F5 IV-V & 4.64 & $3.80^{\mathrm{c}}$ & 0.15 & 0.15 & 9.30 & 6 & $10,6,8,11$ & -0.15 & 0.09 & $6,8,9,11$ \\
\hline HD $142267 \ldots \ldots \ldots \ldots . . .$. & 77801 & 3924 & 5911 & $39 \mathrm{Ser}$ & G0 V & 6.07 & $4.53^{\mathrm{c}}$ & 3.24 & 3.24 & 13.50 & 3 & $17,11,12$ & -0.34 & 0.14 & $3,4,9,11,12,15,16$ \\
\hline HD $147513^{\mathrm{b}} \ldots \ldots \ldots \ldots \ldots$ & 80337 & $620.1 \mathrm{~A}$ & 6094 & $\ldots$ & G5 V & 5.37 & $3.93^{\mathrm{e}}$ & 0.30 & 0.30 & 8.50 & 2 & 10,11 & 0.02 & 0.11 & $3,9,11,14,15,16$ \\
\hline HD $151288 \ldots \ldots \ldots \ldots \ldots$ & 82003 & 638 & $\ldots$ & $\ldots$ & K7 V & 8.10 & 4.71 & $\ldots$ & $\ldots$ & $\ldots$ & 0 & & $\ldots$ & $\cdots$ & \\
\hline HD $154363 \ldots \ldots \ldots \ldots \ldots$ & 83591 & 653 & $\ldots$ & $\ldots$ & $\mathrm{K} 5 \mathrm{~V}$ & 7.70 & 4.73 & $\ldots$ & $\ldots$ & $\ldots$ & 0 & & $\ldots$ & $\ldots$ & \\
\hline HD $156026 \ldots \ldots \ldots \ldots \ldots$ & 84478 & 664 & $\ldots$ & 36 Oph $\mathrm{C}$ & K5 V & 6.33 & $3.47^{\mathrm{c}}$ & 8.80 & 8.64 & 8.96 & 3 & 8 & -0.16 & 0.07 & $3,8,12,15$ \\
\hline HD $157881 \ldots \ldots \ldots \ldots . .$. & 85295 & 673 & $\ldots$ & $\ldots$ & K7 V & 7.54 & $4.14^{\mathrm{e}}$ & 9.34 & $\ldots$ & $\ldots$ & 1 & 8 & 0.00 & 0.35 & 3,6 \\
\hline HD $158633 \ldots \ldots \ldots \ldots \ldots$. & 85235 & 675 & 6518 & $\ldots$ & K0 V & 6.44 & $4.52^{\mathrm{c}}$ & 4.27 & $\ldots$ & $\ldots$ & 1 & 17 & -0.43 & 0.08 & $4,6,9,11,16$ \\
\hline HD $160032 \ldots \ldots \ldots \ldots . . .$. & 86486 & 686 & 6569 & $\lambda$ Ara & F3 IV & 4.76 & $3.83^{\mathrm{c}}$ & 2.44 & 1.85 & 3.30 & 4 & $5,6,9,11$ & -0.29 & 0.06 & $3,5,6,9,11,15$ \\
\hline 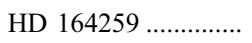 & 88175 & 699 & 6710 & $\zeta$ Ser & F3 V & 4.62 & $3.64^{\mathrm{c}}$ & 1.78 & 1.34 & 2.06 & 5 & $5,6,7,9,11$ & -0.11 & 0.06 & $3,5,6,7,9,11$ \\
\hline HD 165499 .................. & 89042 & 705 & 6761 & $i$ Pav & G1 V & 5.47 & $4.13^{\mathrm{c}}$ & 6.27 & 2.65 & 10.80 & 4 & $6,7,9,11$ & -0.14 & 0.07 & $3,4,6,7,9,11,14$ \\
\hline HD $172051 \ldots \ldots \ldots \ldots \ldots$ & 91438 & 722 & 6998 & $\ldots$ & G5 V & 5.85 & $4.23^{\mathrm{c}}$ & 3.89 & 1.54 & 3.89 & 2 & 17,6 & -0.28 & 0.03 & $4,6,11,14,16$ \\
\hline HD $177565 \ldots \ldots \ldots \ldots \ldots$ & 93858 & 744 & 7232 & $\ldots$ & G8 V & 6.15 & $4.54^{\mathrm{c}}$ & 8.04 & 5.01 & 13.20 & 3 & $6,11,13$ & 0.05 & 0.02 & $3,4,6,11,13,14,15,16$ \\
\hline HD $180617 \ldots \ldots \ldots \ldots \ldots$ & 94761 & $752 \mathrm{~A}$ & $\ldots$ & $\ldots$ & M2.5 & 9.12 & $4.67^{\mathrm{e}}$ & $\ldots$ & $\ldots$ & $\ldots$ & 0 & & $\ldots$ & & \\
\hline HD $182488 \ldots \ldots \ldots \ldots . . . .$. & 95319 & 758 & 7368 & $\ldots$ & G8 V & 6.37 & $4.49^{\mathrm{c}}$ & 4.47 & 4.47 & 10.52 & 2 & 17,6 & 0.11 & 0.08 & $4,6,9,11,14,16$ \\
\hline HD $185395 \ldots \ldots \ldots \ldots \ldots$ & 96441 & $765 \mathrm{~A}$ & 7469 & $\theta$ Cyg & $\mathrm{F} 4 \mathrm{~V}$ & 4.49 & $3.54^{\mathrm{c}}$ & 6.53 & 1.50 & 9.24 & 5 & $8,11,12$ & -0.04 & 0.08 & $3,8,9,11,12,15,16$ \\
\hline HD $187691 \ldots \ldots \ldots \ldots \ldots$. & 97675 & $768.1 \mathrm{~A}$ & 7560 & $o$ Aql & F8 V & 5.12 & $3.90^{\mathrm{c}}$ & 6.61 & 3.70 & 9.00 & 6 & $17,1,2,7,9,11$ & 0.09 & 0.04 & $3,2,7,9,11,15,16$ \\
\hline HD $189245 \ldots \ldots \ldots \ldots . . . .$. & 98470 & 773 & 7631 & $\ldots$ & F7 V & 5.65 & $4.48^{\mathrm{c}}$ & 0.15 & 0.15 & 5.20 & 3 & $10,6,11$ & -0.26 & 0.07 & $6,9,11$ \\
\hline HD $190406 \ldots \ldots \ldots \ldots . . . .$. & 98819 & 779 & 7672 & $15 \mathrm{Sge}$ & G1 V & 5.80 & $4.39^{\mathrm{c}}$ & 2.45 & 2.45 & 8.80 & 4 & $17,1,9,11$ & -0.05 & 0.06 & $3,4,9,11,14,16$ \\
\hline HD 191849 ................. & 99701 & 784 & $\ldots$ & $\ldots$ & M0 V & 7.97 & $4.28^{\mathrm{e}}$ & $\ldots$ & $\ldots$ & $\ldots$ & 0 & & $\ldots$ & $\ldots$ & \\
\hline HD $192310 \ldots \ldots \ldots . . . . .$. & 99825 & 785 & 7722 & $\ldots$ & $\mathrm{K} 0 \mathrm{~V}$ & 5.73 & $3.50^{\mathrm{c}}$ & 8.71 & $\ldots$ & $\ldots$ & 1 & 8 & -0.03 & 0.10 & $3,4,6,11,12,15,16$ \\
\hline HD $196877 \ldots \ldots \ldots \ldots . . . . .$. & 102186 & 798 & $\ldots$ & $\ldots$ & K7 V & 8.83 & 5.47 & $\ldots$ & $\ldots$ & $\ldots$ & 0 & & $\ldots$ & $\ldots$ & \\
\hline HD $198149 \ldots \ldots \ldots \ldots \ldots$ & 102422 & 807 & 7957 & $\eta$ Сер & K0 IV & 3.41 & $1.28^{\mathrm{e}}$ & $\ldots$ & $\ldots$ & $\ldots$ & 0 & & -0.16 & 0.05 & 3 \\
\hline 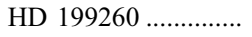 & 103389 & 811 & 8013 & $\ldots$ & F7 V & 5.70 & $4.48^{\mathrm{c}}$ & 3.18 & 2.90 & 3.46 & 2 & 6,11 & -0.20 & 0.12 & $6,9,11$ \\
\hline HD $213845 \ldots \ldots \ldots \ldots . . . .$. & 111449 & 863 & 8592 & $v$ Aqr & F7 V & 5.21 & $4.33^{\mathrm{c}, \mathrm{e}}$ & 0.15 & 0.15 & 2.32 & 4 & $10,6,9,11$ & 0.02 & 0.10 & $6,9,11,15$ \\
\hline HD $215648 \ldots \ldots \ldots \ldots . . . . .$. & 112447 & $872 \mathrm{~A}$ & 8665 & $\xi$ Peg & F7 V & 4.20 & $2.96^{\mathrm{c}}$ & 7.24 & 2.24 & 7.24 & 5 & $17,7,9,11,12$ & -0.27 & 0.09 & $3,7,9,11,12,15,16$ \\
\hline
\end{tabular}


TABLE 1-Continued

\begin{tabular}{|c|c|c|c|c|c|c|c|c|c|c|c|c|c|c|c|}
\hline \multirow[b]{3}{*}{ StAR } & \multirow[b]{3}{*}{ HIP } & \multirow[b]{3}{*}{ GJ } & \multirow[b]{3}{*}{ HR } & \multirow[b]{3}{*}{ OtHER NAME } & \multirow[b]{3}{*}{ SPectral Type } & \multirow{3}{*}{$\begin{array}{c}V \\
(\mathrm{mag})\end{array}$} & \multirow{3}{*}{$\begin{array}{c}K \\
(\mathrm{mag})\end{array}$} & \multicolumn{5}{|c|}{ AgE } & \multirow{2}{*}{\multicolumn{2}{|c|}{$[\mathrm{Fe} / \mathrm{H}]$}} & \multirow[b]{3}{*}{ REFERENCES } \\
\hline & & & & & & & & \multirow{2}{*}{$\begin{array}{c}\mathrm{Mo} / \mathrm{W} / \text { Average }^{\mathrm{a}} \\
\text { (Gyr) }\end{array}$} & \multirow{2}{*}{$\begin{array}{l}\text { Min } \\
\text { (Gyr) }\end{array}$} & \multirow{2}{*}{$\begin{array}{l}\text { Max } \\
(\mathrm{Gyr})\end{array}$} & \multirow{2}{*}{$\begin{array}{l}\text { Number of } \\
\text { Estimates }\end{array}$} & \multirow[b]{2}{*}{ References } & & & \\
\hline & & & & & & & & & & & & & Average & $\sigma$ & \\
\hline HD $217357 \ldots \ldots \ldots \ldots \ldots \ldots$ & 113576 & 884 & $\ldots$ & $\ldots$ & K5 & 7.88 & 4.48 & & $\ldots$ & & 0 & & & & \\
\hline 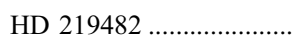 & 114948 & 1282 & 8843 & $\ldots$ & F7 V & 5.64 & $4.44^{\mathrm{c}}$ & 6.07 & 5.60 & 6.54 & 2 & 5,11 & -0.16 & 0.06 & $5,9,11$ \\
\hline 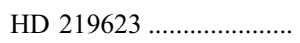 & 114924 & 4324 & 8853 & $\ldots$ & F7 V & 5.58 & $4.31^{\mathrm{c}}$ & 5.06 & 4.60 & 5.50 & 4 & $2,7,9,11$ & -0.05 & 0.09 & $3,2,4,7,9,11,15$ \\
\hline 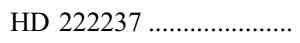 & 116745 & 902 & $\ldots$ & $\ldots$ & $\mathrm{K} 3 \mathrm{~V}$ & 7.09 & 4.58 & $\ldots$ & $\ldots$ & $\ldots$ & 0 & & -0.16 & 0.14 & $4,9,11,15,16$ \\
\hline 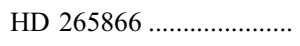 & 33226 & 251 & $\ldots$ & $\ldots$ & M3.5 & 9.89 & 5.28 & $\ldots$ & $\ldots$ & $\ldots$ & 0 & & $\ldots$ & $\ldots$ & \\
\hline \multicolumn{16}{|c|}{ Companions } \\
\hline HD $10360 \mathrm{~J} \ldots \ldots \ldots \ldots \ldots \ldots \ldots$ & 7751 & 66 & $\ldots$ & p Eri & K0 V & 5.07 & $3.51^{\mathrm{c}}$ & & $\ldots$ & $\ldots$ & 0 & & -0.28 & 0.00 & 4,15 \\
\hline 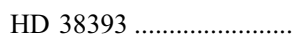 & 27072 & $216 \mathrm{~A}$ & 1983 & $\gamma$ Lep & F7 V & 3.59 & $2.42^{\mathrm{e}}$ & 0.30 & 0.30 & 9.51 & 6 & $10,2,7,8$ & -0.08 & 0.05 & $3,2,4,7,8,9,11,12,15$ \\
\hline HD 50281B & $\ldots$ & $250 \mathrm{~B}$ & $\ldots$ & $\ldots$ & M2 & 10.10 & 5.72 & $\ldots$ & $\ldots$ & $\ldots$ & 0 & & $\ldots$ & $\ldots$ & \\
\hline 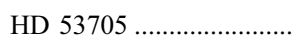 & 34065 & $264.1 \mathrm{~A}$ & 2667 & $\ldots$ & G3 V & 5.56 & $4.04^{\mathrm{c}}$ & 12.90 & $\ldots$ & $\ldots$ & 1 & 11 & -0.27 & 0.06 & $3,4,11,14,15,16$ \\
\hline 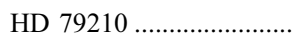 & 45343 & $338 \mathrm{~A}$ & $\ldots$ & $\ldots$ & K7 & 7.64 & 3.99 & $\ldots$ & $\ldots$ & $\ldots$ & 0 & & $\ldots$ & $\ldots$ & \\
\hline HD $131976 \ldots \ldots \ldots \ldots$ & 73182 & $570 \mathrm{~B}$ & $\ldots$ & & M1 V & 8.01 & $3.90^{\mathrm{e}}$ & & $\ldots$ & & 0 & & & & \\
\hline
\end{tabular}

Notes.-Spectral types from SIMBAD. Visual magnitudes are as quoted in SIMBAD, typically from the Hipparcos satellite; $K$ magnitudes are from 2MASS unless otherwise noted.

a Age from Montes's paper, then from Wright's paper if available; otherwise, an average of literature values.

${ }^{\mathrm{b}}$ Known planet-bearing star.

Star has one or more bad 2MASS values (err $>20 \%$ ).

d Star has a wide binary companion that was included in the survey, as listed at the bottom of the table

Star has $J H K$ values from Johnson or other literature.

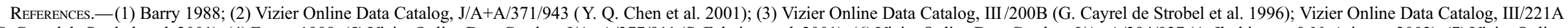

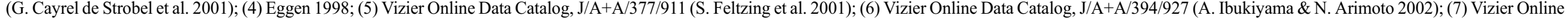

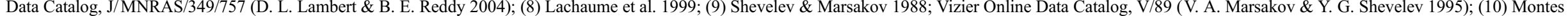

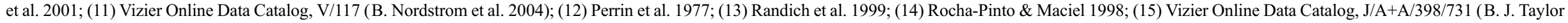
2002); (16) Valenti \& Fischer 2005; (17) Wright et al. 2004 
TABLE 2

Measured and Predicted Flux Densities at 24 and $70 \mu \mathrm{m}$ (in mJy)

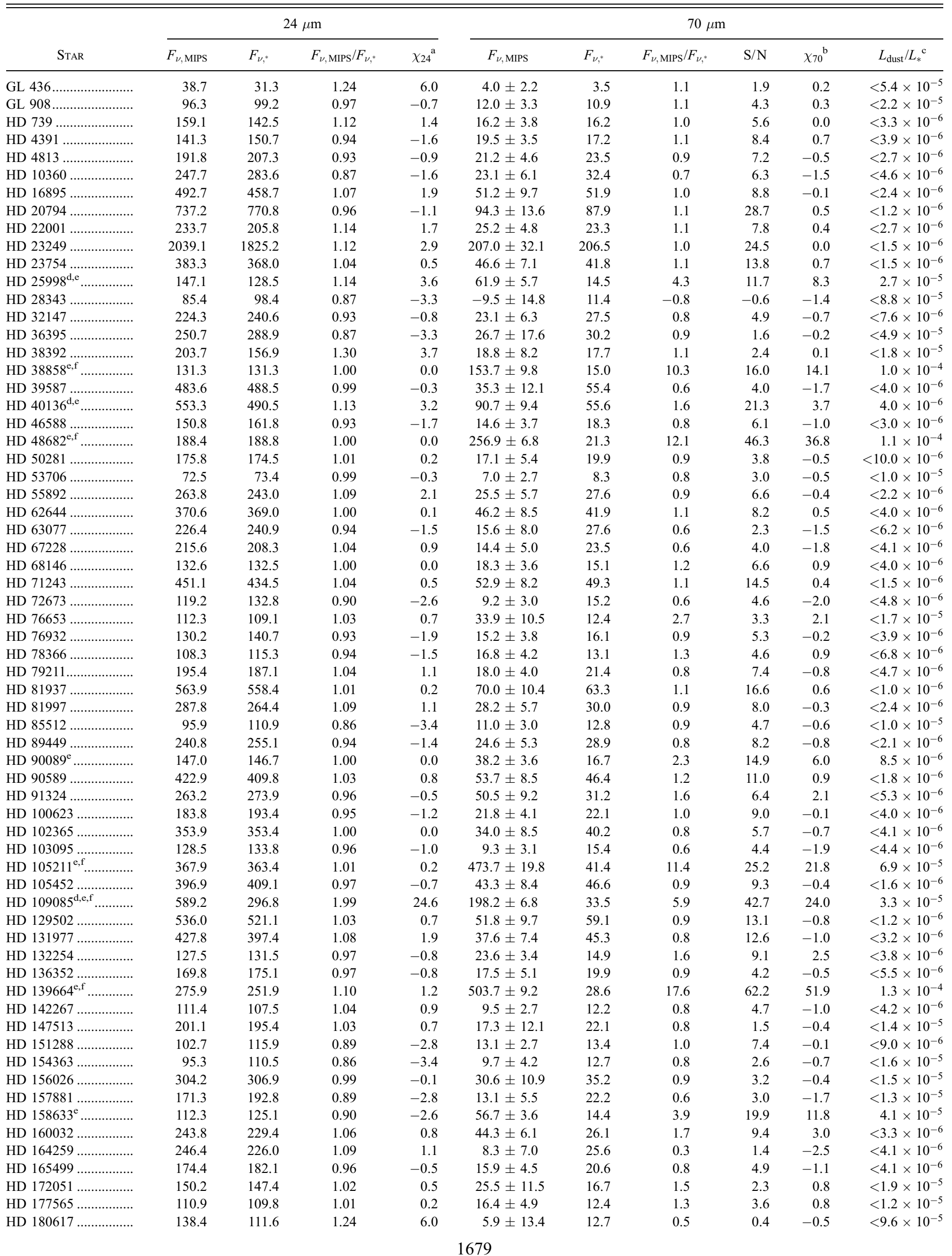


TABLE 2-Continued

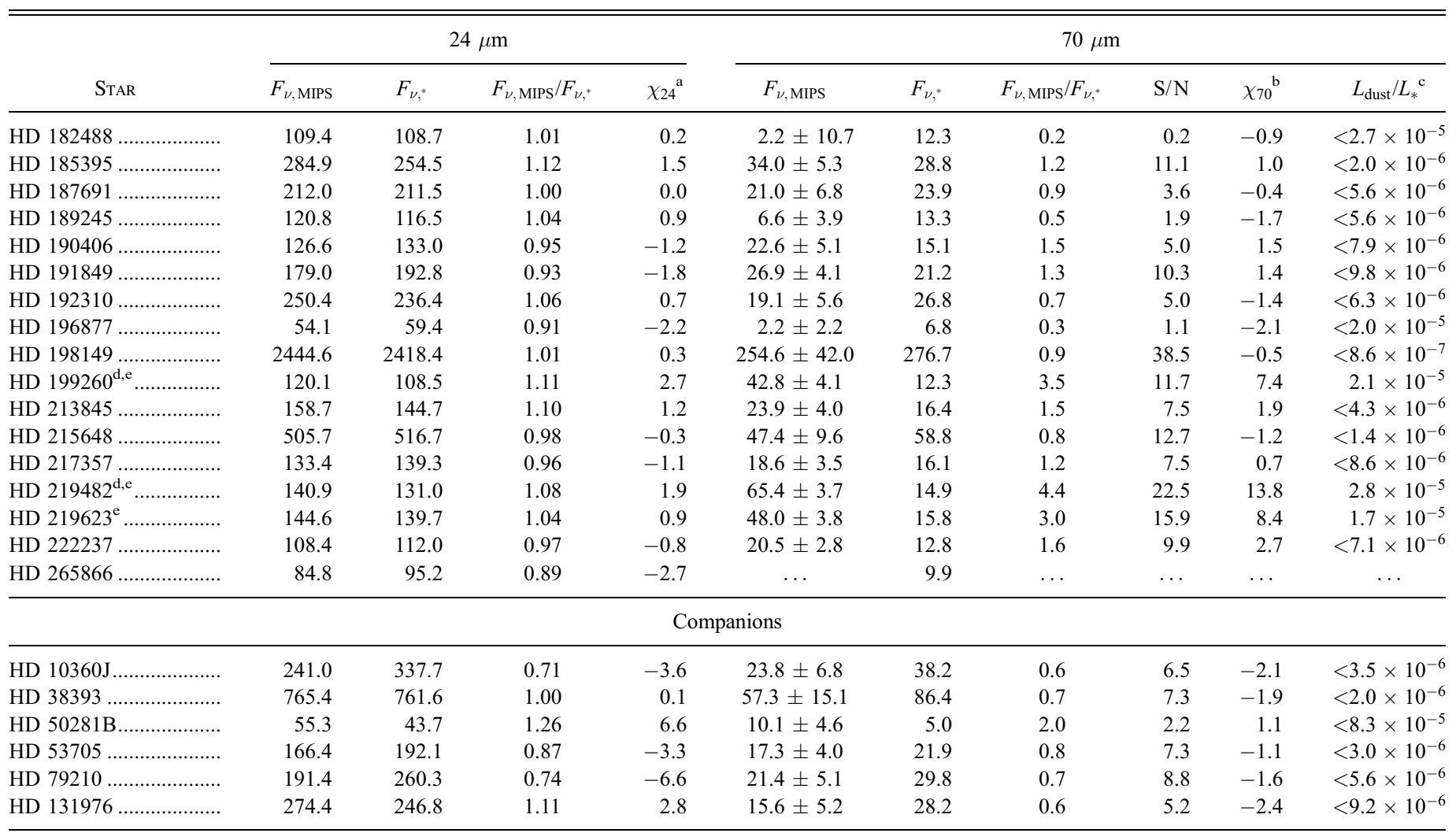

${ }^{a}$ Significance of $24 \mu \mathrm{m}$ excess (eq. [1]).

${ }^{\mathrm{b}}$ Significance of $70 \mu \mathrm{m}$ excess (eq. [2]).

${ }^{c}$ Minimum $L_{\text {dust }} / L_{*}$ from $70 \mu \mathrm{m}$ emission (eq. [3]).

${ }^{\mathrm{d}}$ Star with excess $24 \mu \mathrm{m}$ emission.

e Star with excess $70 \mu \mathrm{m}$ emission.

${ }^{\mathrm{f}}$ Star with resolved $70 \mu \mathrm{m}$ emission.

\subsection{Data Reduction}

\subsubsection{MIPS Observations}

Overall, our data analysis is similar to that previously described in Beichman et al. (2005a) and Bryden et al. (2006). At

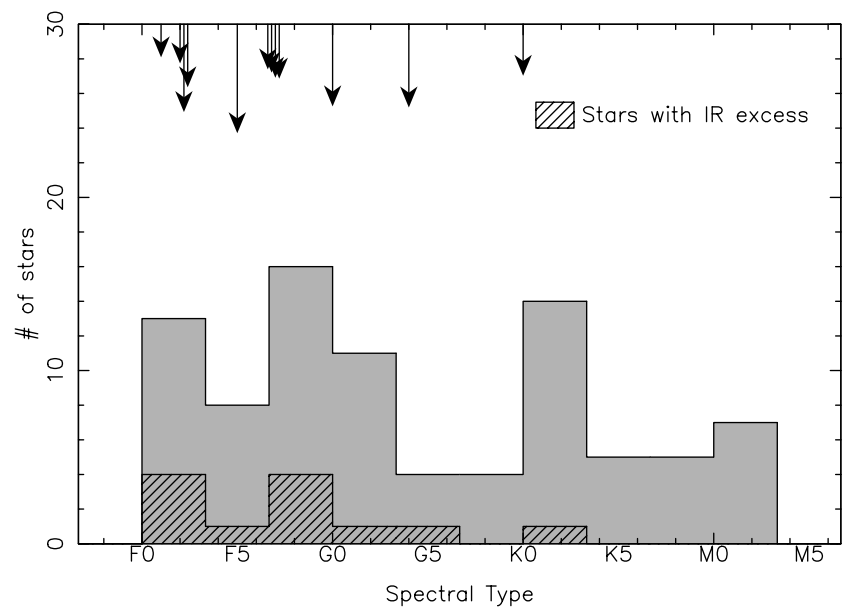

FIG. 1.-Spectral type distribution for stars in this SIM PlanetQuest/TPF sample. The spectral types of stars found to have $70 \mu \mathrm{m}$ excess are highlighted within the histogram (hatched) and are individually flagged with arrows at the top of the plot. The length of each arrow is an indicator of the strength of $70 \mu \mathrm{m} \mathrm{ex}-$ cess relative to the stellar photosphere. We find that $70 \mu \mathrm{m}$ excess is more readily detected around early-type stars.
$24 \mu \mathrm{m}$, images were created from the raw data using the DAT software developed by the MIPS instrument team (Gordon et al. 2005). At $70 \mu \mathrm{m}$, images were processed beyond the standard DAT software to correct for time-dependent transients, corrections that can significantly improve the sensitivity of the measurements

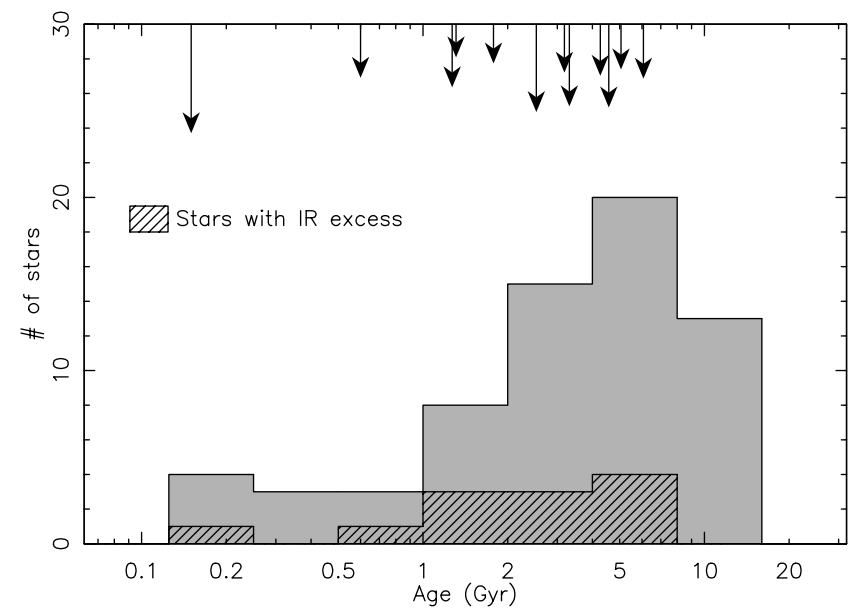

FIG. 2.-Age distribution for stars in this SIM PlanetQuest/TPF sample. The ages of stars with $70 \mu \mathrm{m}$ excess are highlighted within the histogram (hatched) and are individually flagged with arrows at the top of the plot. The length of each arrow is an indicator of the strength of $70 \mu \mathrm{m}$ excess. There is a weak correlation between the detection of IR excess and the stellar age, with no stars older than 7 Gyr having excess emission. 


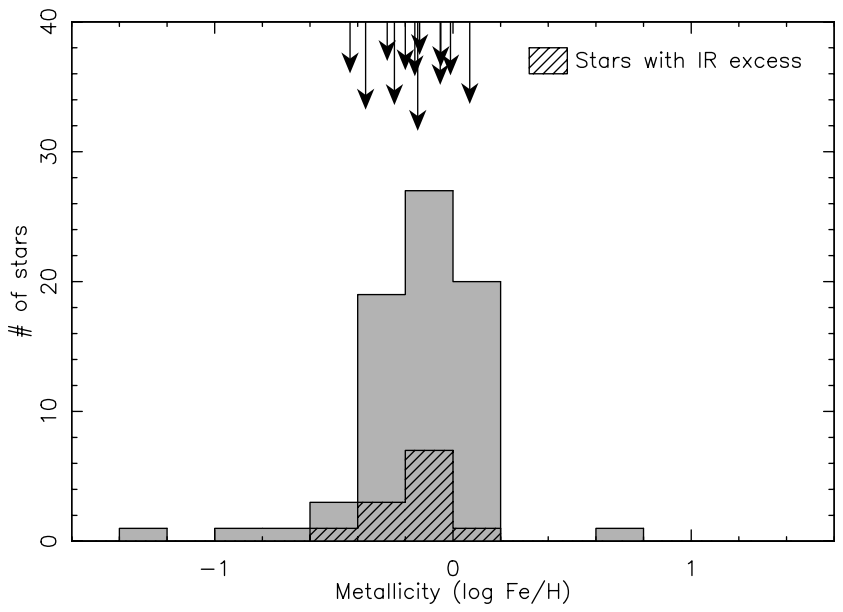

FIG. 3.-Metallicity distribution for stars in this SIM PlanetQuest/TPF sample. The ages of stars with $70 \mu \mathrm{m}$ excess are highlighted within the histogram (hatched) and are individually flagged with arrows at the top of the plot. The length of each arrow is an indicator of the strength of $70 \mu \mathrm{m}$ excess relative to the stellar photosphere. There is no correlation between metallicity and the detection of IR excess.

(Gordon et al. 2004). For both wavelengths, aperture photometry was performed using apertures sizes, background annuli, aperture corrections, and instrument calibration as in Beichman et al. (2005a). We find that the target locations in the $24 \mu \mathrm{m}$ images are consistent with the telescope pointing accuracy of $<1^{\prime \prime}$ (Werner et al. 2004). As such, we use the $24 \mu \mathrm{m}$ centroid as the target coordinates for both wavelengths. Special consideration is made for the six resolved binaries in our sample. Instead of our standard method of aperture photometry with a surrounding sky annulus, the emission at the two stars' locations is fitted with the instrument's point-spread function (PSF). We find that for binary stars with small angular separations, simultaneously fitting their overlapping PSFs results in much improved photometric accuracy. The agreement between PSF fitting and aperture photometry (with appropriate aperture correction) for isolated stars is excellent (Gordon et al. 2005). For all of the stars, the MIPS flux and noise measurements are listed in Table 2.

\subsubsection{IRAC Observations}

The IRAC subarray images of the four M stars were reduced following the technique described by the FEPS Legacy Team (J. Carpenter et al. 2006, in preparation). The pixel sizes are corrected for distortion and a pixel-phase correction is made to channel 1. Stellar fluxes are measured within an aperture of 10 pixels $\left(=12^{\prime \prime}\right)$, with a background annulus from 10 to 20 pixels. The photometric measurements for each star at the four IRAC wavelengths are listed in Table 3.

\subsection{Photospheric Extrapolations and Limits on $24 \mu \mathrm{m}$ Excess}

To determine whether any of our target stars have an IR excess, we compare the measured photometry against predicted photo-

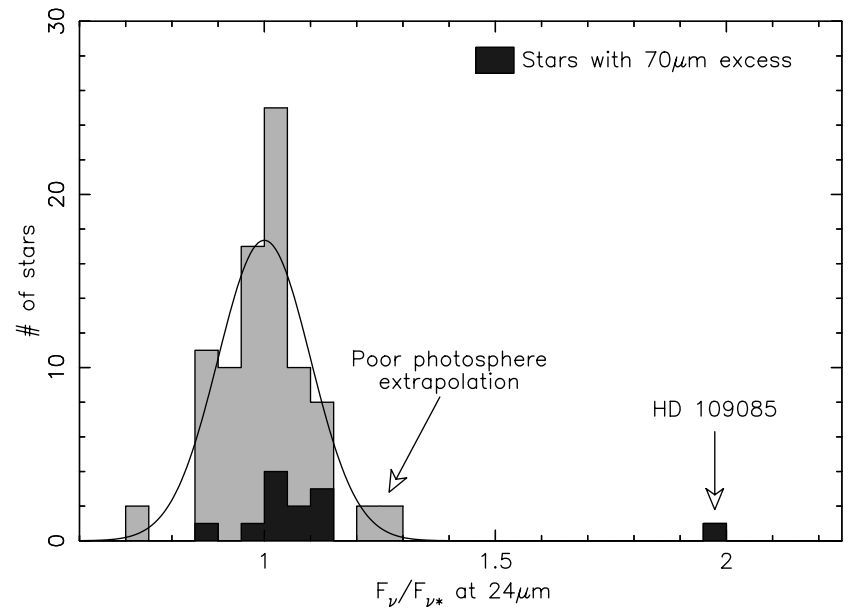

FIG. 4.-Distribution of $24 \mu \mathrm{m}$ fluxes relative to the expected photospheric values. A Gaussian distribution with $10 \%$ dispersion (solid curve) is shown for comparison. One star (HD 109085, a star previously identified as having excess emission) clearly stands out from the main population. The broad dispersion within this population is due to a variety of factors. Some stars have poor estimates of the stellar flux at $24 \mu \mathrm{m}$ due to poor near-IR data or photospheric models, particularly for the set of late $\mathrm{K}$ and $\mathrm{M}$ stars marked "poor extrapolation" in the figure. The spread of values is also increased by sources with true, weak excesses (at the level of $\sim 10 \%$ above the stellar photosphere). Stars with excesses at a longer wavelength $(70 \mu \mathrm{m})$ are shown with black shading.

spheric levels. A detailed description of our stellar atmosphere fitting, as applied to F5-K5 stars, is presented in the Appendix of Bryden et al. (2006). The stars observed here, however, span a greater range of spectral types than previously considered. In particular, our sample contains late K- and M-type stars with numerous broad molecular features for which the stellar models (Kurucz 2003) begin to lose their accuracy.

This accuracy can be directly assessed by examining how well the observed flux levels match those predicted. We use the ratio, $F_{\text {MIPS }} / F_{\text {photosphere }}$, to assess the photospheric extrapolation using the fact previously established in Bryden et al. (2006), Beichman et al. (2006), and earlier references cited therein that excesses at $24 \mu \mathrm{m}$ are rare $(\sim 1 \%)$. Figure 4 shows the distribution of this ratio. After excluding one outlying star with a strong 24 and $70 \mu \mathrm{m}$ excess (HD 109085), the 88 flux measurements at $24 \mu \mathrm{m}$ have

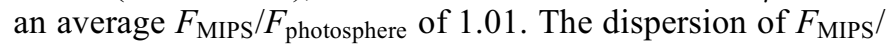
$F_{\text {photosphere }}$ in Figure 4 is 0.10 , which is relatively large compared to the previous result for just F5-K5 stars (0.07; Bryden et al. 2006). We identify three causes for this larger dispersion.

\subsubsection{Quality of Near-IR Photometry}

The SIM PlanetQuest/TPF sample contains a number of nearby stars that are brighter than the stars in the FGK survey. Stars brighter than about $K_{s}=4$ mag have saturated Two Micron All Sky Survey (2MASS) measurements resulting in large photometric uncertainties $(\sim 0.25 \mathrm{mag})$. For several of these stars,

TABLE 3

IRAC ObSERVATIONS OF M STARS

\begin{tabular}{|c|c|c|c|c|c|}
\hline 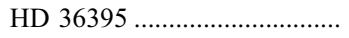 & M1.5 V & 7.64 & 5.62 & 3.74 & 2.22 \\
\hline 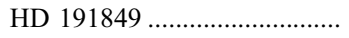 & $\mathrm{K} 7 / \mathrm{M} 0$ & 6.46 & 4.12 & 2.70 & 1.58 \\
\hline 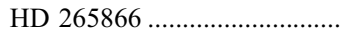 & M3.5 & 2.75 & 1.83 & 1.22 & 0.70 \\
\hline
\end{tabular}

NotE.-Flux density uncertainties are dominated by calibration uncertainties, typically $5 \%$ for the IRAC bands. 
particularly the early F-type stars that are the brightest in the sample, Johnson $K$-band photometry is available in the literature with much better accuracy $(\sim 0.05 \mathrm{mag})$ than the saturated $2 \mathrm{MASS}$ values, but worse than the best 2 MASS values $(\sim 0.03 \mathrm{mag})$. In such cases, the saturated 2MASS values are supplanted by the better data. However, the uncertainty in the near-IR photometry for the remaining 2MASS-saturated stars causes difficulty in extrapolating to longer wavelengths and results in a greater dispersion than when only stars with high-quality 2 MASS data are used, $\sigma\left(F_{\text {MIPS }} / F_{\text {photosphere }}\right)=0.09$ versus 0.07 for types F5-K5 .

\subsubsection{Intrinsic Variability}

Stellar variability between the epochs of the Spitzer data and the photometry used to estimate the photospheric contribution could account for some of the dispersion in $F_{\text {MIPS }} / F_{\text {photosphere }}$ To investigate this possibility, we examined the Hipparcos photometry for the 66 stars of our sample for which these data are available (Perryman et al. 1997). Only three stars (GL 436, HD 79211, and HD 265866) showed a scatter in $\operatorname{mag}(\mathrm{Hp})$ in excess of $0.02 \mathrm{mag}$, while the vast majority had scatter less than $0.01 \mathrm{mag}$. In particular, none of the 10 stars younger than 1 Gyr and thus possibly more variable than the rest of the sample showed variability above this level. GL 436 and HD 265866 are faint $V \sim 10$ mag $\mathrm{M}$ stars so that the level of Hipparcos scatter is not significant. The large scatter for HD 79211 (0.23 mag) is due to multiplicity and is also not significant. As discussed below, one non-Hipparcos star, HD 38392, shows a low level of variability and a correspondingly larger deviation in $F_{\text {MIPS }} / F_{\text {photosphere }}$.

\subsubsection{Quality of Photospheric Models}

The ability to extrapolate from visible and near-IR photometry to MIPS wavelengths appears to be an issue for spectral types later than the F5-K5 range used in the FGK survey. For all stars with accurate $2 \mathrm{MASS}$ data, Figure 5 plots the directly observable $K_{s}-[24]$ color, a quantity independent of the stellar atmosphere models. With the exception of the $\mathrm{M}$ stars, all of the averages are consistent with a constant color of $K_{s}-[24] \simeq 0.02 \pm 0.02$. Most interestingly, an apparently abrupt transition occurs between the late K stars and M stars, with the average $K_{s}-[24]$ color jumping up $\sim 0.4$ mag for the cooler stars. This trend of redder $K_{s}-[24]$ for later spectral types was first noticed by T. N. Gautier et al. (2006, in preparation), whose $\mathrm{M}$ star data are shown for comparison.

We next consider the ratio of the observed flux at $24 \mu \mathrm{m}$ to that predicted by photospheric models ( $\left.F_{\text {MIPS }} / F_{\text {photosphere }}\right)$ as a function of spectral type. Solar-like stars (types F5-K4) have an overall average of $F_{\text {MIPS }} / F_{\text {photosphere }}=0.98 \pm 0.01$ with a dispersion of $5 \%$ among the stars with good 2 MASS data and excluding stars with excess emission at $70 \mu \mathrm{m}$ (identified in $\S 3.3$ ). For F0-F4 stars, the observed fluxes are marginally higher than those predicted, with an average $F_{\text {MIPS }} / F_{\text {photosphere }}=1.03 \pm 0.02$. For late $\mathrm{K}$ stars with good 2MASS observations the observed fluxes are consistently below expectation with an average $F_{\text {MIPS }} / F_{\text {photosphere }}$ of $0.87 \pm 0.03$. Since the observed $K-[24]$ color is flat (Fig. 5), this offset is likely a fault of the photospheric modeling or of our fitting procedure. Not surprisingly, the models have the greatest difficulty with the M stars, which have average $F_{\text {MIPS }} / F_{\text {photosphere }}=$ $1.16 \pm 0.06$. This difference between measured and predicted fluxes for the M stars remains even if NextGen (Hauschildt et al. 1999) models are used instead of Kurucz models. However, with an accurate determination of each star's effective temperature and with more advanced stellar models (PHOENIX; Brott \& Hauschildt 2005), T. N. Gautier et al. (2006, in preparation) were able to fit the $24 \mu \mathrm{m}$ colors of $\mathrm{M}$ stars.

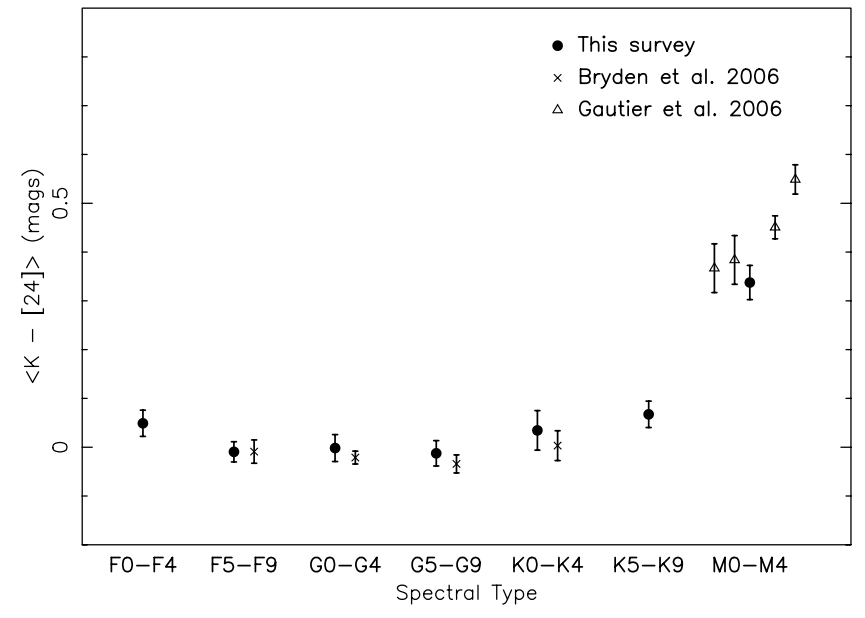

FIG. 5.-Average $24 \mu \mathrm{m}$ color relative to $2 \mathrm{MASS} K_{s}$ band $(2.16 \mu \mathrm{m})$ as a function of spectral type. Stars with excess emission or with poor $K_{s}$ measurements are excluded. Error bars indicate the error on the mean value within each bin (not the overall dispersion). Stellar colors from the Bryden et al. (2006) F5-K5 survey and the T. N. Gautier et al. (2006, in preparation) M star survey are also shown for comparison. The trend is relatively flat over most of the range, with significantly red colors only seen among the M-type stars.

Thus, knowing that these trends in $F_{\text {MIPS }} / F_{\text {photosphere }}$ exist and may ultimately be explained with better modeling, we can compare each star with the average $K_{s}-[24]$ color within its spectral type bin (Fig. 5) to look for dust excesses. With this methodology, we find no evidence for a $24 \mu \mathrm{m}$ excess toward any of our M stars. This negative result is strengthened when IRAC photometry is available. For the four M stars with IRAC data (Table 3), the inclusion of 3.5-8.0 $\mu \mathrm{m}$ fluxes into the fit modifies the average of $F_{\text {MIPS }} / F_{\text {photosphere }}$ from 1.32 with a dispersion of 0.21 to $F_{\text {MIPS }} /$ $F_{\text {photosphere }}=0.92$ with a dispersion of 0.04 , confirming that the M stars' $24 \mu \mathrm{m}$ fluxes are consistent with emission from the stellar photosphere alone.

\subsubsection{Presence of a Weak Excess at $24 \mu \mathrm{m}$}

Finally, the third reason for increased dispersion in the $F_{\text {MIPS }} /$ $F_{\text {photosphere }}$ values, in addition to poor near-IR photometry and less accurate stellar photospheres or model fitting for late-type stars, is the presence of weak but real excess emission from dust toward some stars. In $\S 3.3$ we identify some of our target stars as having strong excess emission at $70 \mu \mathrm{m}$. Only one of these objects, HD $109085^{12}$ (the labeled value in Fig. 4), also has an immediately obvious IR excess at $24 \mu \mathrm{m}$.

Taken in composite, however, the stars with $70 \mu \mathrm{m}$ excesses tend to have a weak $24 \mu \mathrm{m}$ excess. Considering only F0-K 5 stars with good near-IR photometry, those with $70 \mu \mathrm{m}$ excess have an

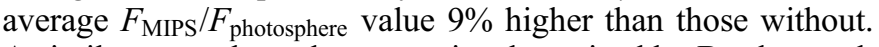
A similar general trend was previously noticed by Bryden et al. (2006) and was confirmed in the IRS spectra of individual objects with $70 \mu \mathrm{m}$ excess, which tend to rise above the stellar photosphere longward of $25 \mu \mathrm{m}$ (Beichman et al. 2006). Combining the F0-K5 stars in this sample with those from Bryden et al. (2006), we are no longer limited by small number statistics and

\footnotetext{
12 An excess was first detected around HD 109085 ( $=\eta$ Crv) by IRAS (Aumann 1988; Stencel \& Backman 1991) and subsequently with SCUBA at submillimeter wavelengths (Sheret et al. 2004; Wyatt et al. 2005). Consistency between the IRAS flux at $25 \mu \mathrm{m}$ and the MIPS $24 \mu \mathrm{m}$ flux measured here depends strongly on the application of color corrections, which are functions of the assumed dust temperature. For dust temperatures around $200-400 \mathrm{~K}$ and assuming the photospheric value given in Table 2, we find good consistency between the two measurements.
} 
the correlation between 70 and $24 \mu \mathrm{m}$ excess becomes significant at the $3 \sigma$ level. The average $24 \mu \mathrm{m}$ excess for stars with $70 \mu \mathrm{m}$ excess is $0.079 \pm 0.026$ times the stellar flux.

To assess the significance of a possible $24 \mu \mathrm{m}$ excess on a starby-star basis, we define the parameter $\chi_{24}$, which corresponds to the $n \sigma$ significance of any deviation from the expected photospheric value:

$$
\chi_{24} \equiv \frac{F_{24}-F_{*}}{\sigma_{24}},
$$

where $F_{24}$ is the measured flux, $F_{*}$ is the expected stellar flux, and $\sigma_{24}$ is the noise level, all at $24 \mu \mathrm{m}$. A similar definition follows for $70 \mu \mathrm{m}$ (eq. [2]). We take the noise to be the larger of either $4 \%$ for sources with good 2MASS or Johnson data or $8 \%$ for sources with poor near-IR photometry. These values are based on the dispersions in $F_{\mathrm{MIPS}} / F_{\text {photosphere }}$ for the stars without $70 \mu \mathrm{m}$ excesses. Ignoring the previously discussed late $\mathrm{K}$ and $\mathrm{M}$ stars with poor photospheric extrapolations, we find that the deviations from photospheric emission skew sharply to positive values for stars with $70 \mu \mathrm{m}$ excess (Fig. 4, black shading). Using this analysis, we identify statistically significant $24 \mu \mathrm{m}$ excesses accompanying a stronger $70 \mu \mathrm{m}$ excess around two stars, HD 25998 $(3.6 \sigma)$ and HD 40136 (3.2 $\sigma$ ), in addition to HD 109085 discussed earlier. At slightly lower significance we find hints of a $24 \mu \mathrm{m}$ excess for HD $199260(2.7 \sigma)$ and HD $219482(1.9 \sigma)$, which the accompanying $70 \mu \mathrm{m}$ excess suggests could be real.

A number of other stars show strong deviations from photospheric values without an accompanying $70 \mu \mathrm{m}$ excess: the deviant $F_{\text {MIPS }} / F_{\text {photosphere }}$ values of the M stars have already been discussed and attributed to poor photospheric extrapolation; HD 38392 has an apparent $30 \%$ excess at $24 \mu \mathrm{m}$, which we attribute to the difficulty of obtaining accurate measurements due to (1) saturated 2MASS measurements, (2) proximity to a nearby, bright companion (HD 38393), and (3) the possible variability of the star itself at the $5 \%$ peak-to-peak level (Nitschelm et al. 2000). Finally, HD 23249 and HD 55892 have $2<\chi_{24}<3$ and $\chi_{70}<2$. These deviations could simply be statistical fluctuations or they could be hints of an excess like that seen toward HD 69830, which is prominent only in the 8-34 $\mu \mathrm{m}$ region but not at $70 \mu \mathrm{m}$ (Beichman et al. 2005b). Without additional data, e.g., IRS spectra, we cannot assess the reality of the excesses around these last two stars.

\subsection{Detection of $70 \mu \mathrm{m}$ Excess}

Having used $24 \mu \mathrm{m}$ fluxes to test the accuracy of our stellar photosphere predictions, we next consider the frequency and strength of excess emission at $70 \mu \mathrm{m}$. The distribution of $70 \mu \mathrm{m}$ flux densities relative to the expected photospheric values is shown in Figure 6. Unlike the tight distribution of flux ratios at $24 \mu \mathrm{m}$, many stars have $70 \mu \mathrm{m}$ flux densities much higher than expected from the stellar photosphere alone. In several cases, the flux is more than an order of magnitude greater than expectation. Twelve of these stars will be identified in the following as having statistically significant IR excess. Excluding these stars with excesses and those with signal-to-noise ratio $(\mathrm{S} / \mathrm{N})<3$, the average ratio of MIPS flux to predicted photosphere is $\left|F_{\text {MIPS }} / F_{\text {photosphere }}\right|=$ $1.02 \pm 0.05$, consistent with the overall calibration.

The dispersion in the $70 \mu \mathrm{m}$ data is $\sim 40 \%$ (excluding the stars with excesses), considerably higher than that in the $24 \mu \mathrm{m}$ data. An analysis of the noise levels in each individual field is required to assess whether the IR excesses are statistically significant. Many contributions to the overall error budget must be considered, including those arising from stellar photosphere modeling, instrument calibration, sky background variation, and photon de-

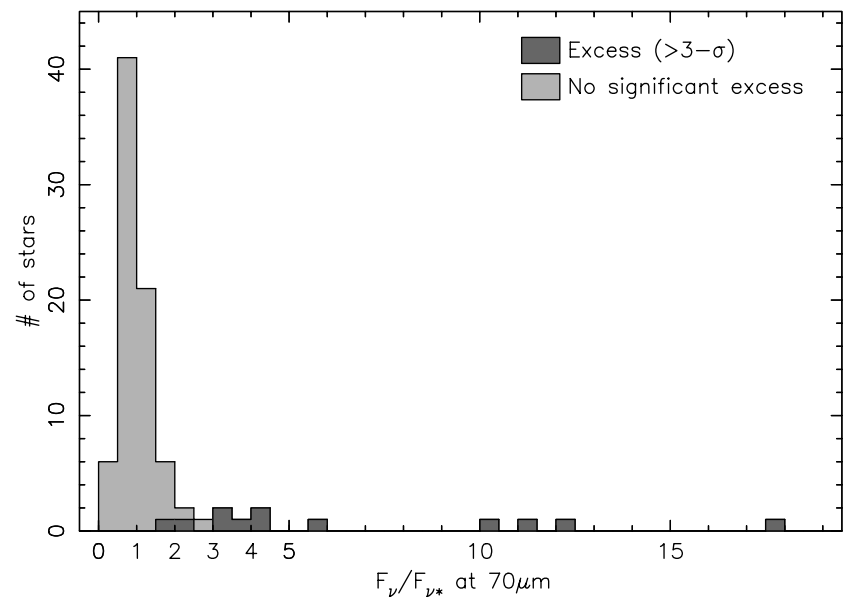

FIG. 6.-Distribution of $70 \mu \mathrm{m}$ fluxes relative to the expected photospheric values. While most stars cluster around unity (consistent with emission from the star alone), many show a high degree of excess emission attributable to circumstellar dust.

tector noise. At $70 \mu \mathrm{m}$, the calibration uncertainty and the background noise within each image are considerably larger than at $24 \mu \mathrm{m}$. On top of an assumed calibration uncertainty of $15 \%$, we directly measure the standard deviation of the background flux when each field is convolved with our chosen aperture size. This background noise, which ranges from $\sim 2$ to $20 \mathrm{mJy}$ with a median of $3.7 \mathrm{mJy}$, is due primarily to extragalactic source confusion and cirrus contamination, rather than photon noise, and hence cannot be greatly reduced by additional integration time (for a more detailed analysis of the $70 \mu \mathrm{m}$ noise levels, see Bryden et al. 2006). Based on this measured background noise, we determine the $\mathrm{S} / \mathrm{N}$ for each star, as listed in Table 2. Despite the high level of noise in some fields due to cirrus contamination and/or background galaxies, 72 out of the 87 stars in our sample with $70 \mu \mathrm{m}$ data are detected with $\mathrm{S} / \mathrm{N}$ greater than 3 . The median $\mathrm{S} / \mathrm{N}$ for all of our target stars is 6.6, excluding the sources identified as having excess emission (which have a median $\mathrm{S} / \mathrm{N}$ of over 20 ).

Adding both background noise and calibration error together gives us a total noise estimate for each $70 \mu \mathrm{m}$ target. In Table 2 we list these noise levels, along with the measured and photospheric fluxes, for each observed star. We use these noise estimates to calculate $\chi_{70}$, which corresponds to the $n \sigma$ significance of any deviation from the expected level of photospheric emission:

$$
\chi_{70} \equiv \frac{F_{70}-F_{*}}{\sigma_{70}},
$$

where $F_{70}$ is the measured flux, $F_{*}$ is the expected stellar flux, and $\sigma_{70}$ is the noise level, all at $70 \mu \mathrm{m}$. Based on this criterion, we find that 12 out of 88 stars have a $3 \sigma$ or greater excess at $70 \mu \mathrm{m}$ : HD 25998, HD 38858, HD 40136, HD 48682, HD 90089, HD 105211, HD 109085, HD 139664, HD 158633, HD 199260, HD 219482, and HD 219623. In a sample of 88 stars there should be fewer than 1 star with a spurious excess on purely statistical grounds. Although cirrus or extragalactic confusion could produce spurious excesses, careful examination of each of the $70 \mu \mathrm{m}$ images suggests that this is unlikely in the vast majority of cases. For example, the $70 \mu \mathrm{m}$ emission is well centered on the $24 \mu \mathrm{m}$ positions, typically within $\sim 1^{\prime \prime}$. A number of weak excesses could have escaped detection under these criteria. Observations at higher sensitivity or at higher spatial resolution will be needed to identify these. The detection rate of $70 \mu \mathrm{m}$ excess within this sample is $14 \% \pm 4 \%$; combined with the sample of Bryden et al. (2006), this gives an overall detection rate of $13 \% \pm 3 \%$ for cool stars. 


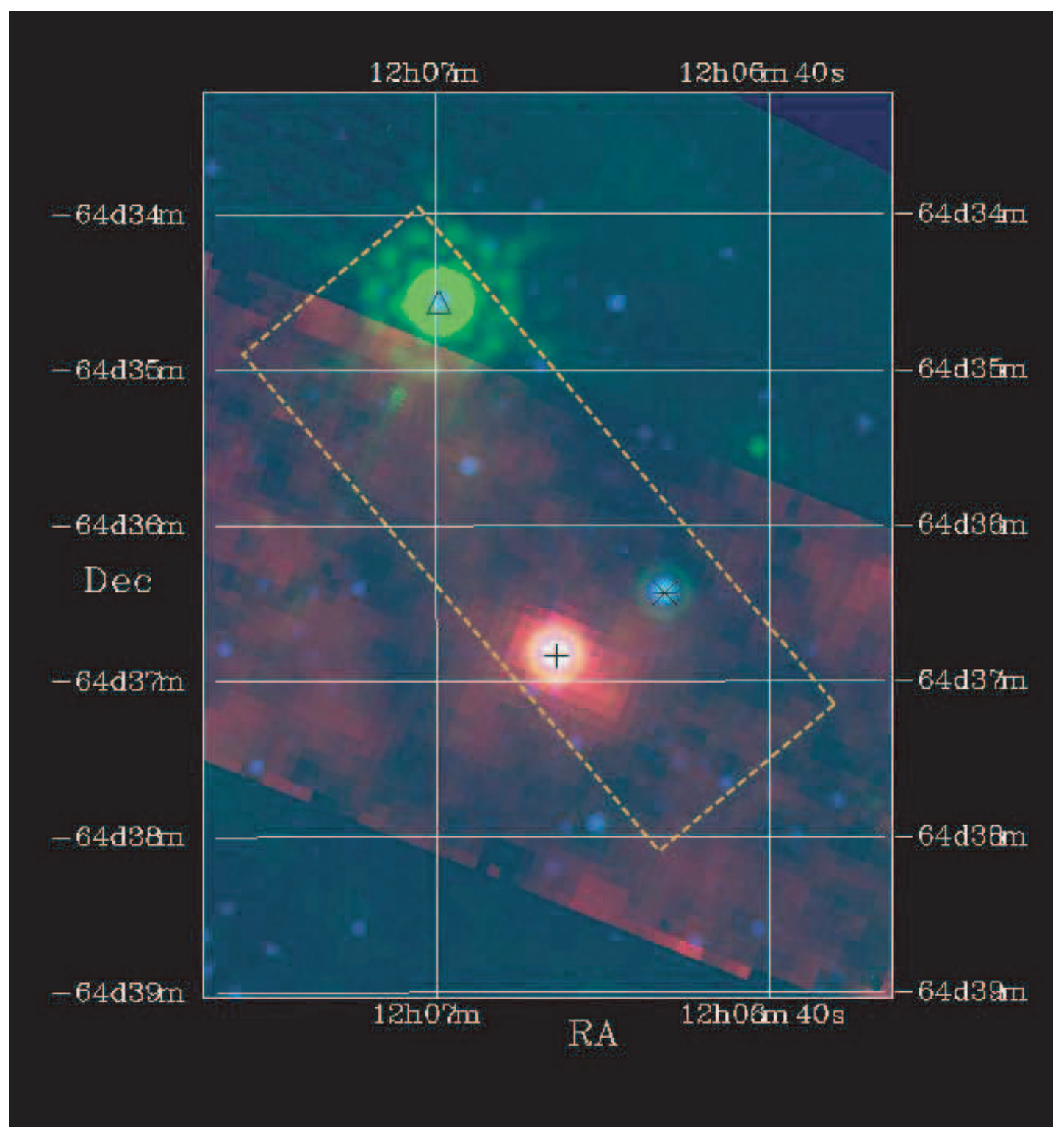

FIG. 7. - Composite image of the field surrounding HD 105211 (marked by a plus sign). In addition to our MIPS $24 \mu \mathrm{m}$ ( green) and $70 \mu \mathrm{m}$ (red) images, the $2 \mathrm{MASS}$ $K_{s}$ band image is overlaid in blue. While dim background stars show up as blue points, the cool Mira variable star CL Cru (triangle) has strong $24 \mu \mathrm{m}$ emission with a much broader PSF, resulting in an overall green color. This bright star contaminated the broad IRAS scanning beam (dashed yellow rectangle), prohibiting the detection of dust around HD 105211. In the MIPS image, HD 105211 is well resolved, showing strong excess emission at $70 \mu \mathrm{m}$. The neighboring star CPD -63 2145B (asterisk), while detected at $24 \mu \mathrm{m}$, does not give off significant emission at $70 \mu \mathrm{m}$.

Four of these stars have been previously identified as having excess emission: HD 40136 ( $=\eta$ Lep; Aumann 1988; Mannings \& Barlow 1998), HD 48682 ( $=\psi 5$ Aur; Aumann \& Probst 1991; Sheret et al. 2004), HD 109085 ( $=\eta$ Crv; Aumann 1988; Wyatt et al. 2005), and HD 139664 (=g Lup; Walker \& Wolstencroft 1988; Habing et al. 1996; Kalas et al. 2006). The eight newly discovered IR-excess stars mostly have $70 \mu \mathrm{m}$ fluxes less than $100 \mathrm{mJy}$, too dim to have been be detected by IRAS. The notable exception is HD 105211, which has a very strong $70 \mu \mathrm{m}$ flux ( 500 mJy) but lies near a bright infrared source (CL Cru); with a separation of 2.'4, this source is easily resolved in the MIPS image (Fig. 7) but still contaminates the large IRAS beam. For the stars without any significant excess emission, $3 \sigma$ upper limits on possible excess flux typically range from 0.2 to 1.0 times the stellar flux, with a median upper limit of $0.6 F_{*}$.

Although the telescope resolution at $70 \mu \mathrm{m}$ is relatively poor (FWHM of $\sim 17^{\prime \prime}$ ), several of these sources appear to be slightly extended in the MIPS images (marked with footnote $\mathrm{f}$ in Table 2). As discussed in a separate paper (G. Bryden et al. 2006, in preparation), examination of the images of these marginally resolved sources does not indicate contamination by background objects, e.g., cirrus or galaxies, but rather that the objects possess truly extended disks. For one of the five Spitzer-resolved disks, HD 139664 , a Hubble Space Telescope (HST) image shows the same orientation of the disk in the visible as in the infrared (Kalas et al. 2006). At distances of 10-20 pc, the resolved disks have apparent radii of hundreds of AU. As discussed below, maintaining a temperature of $\sim 50 \mathrm{~K}$ at these distances (warm enough to emit strongly at $70 \mu \mathrm{m}$ ) requires relatively small grains with low emissivities.

\section{PROPERTIES OF THE DETECTED DUST}

Beyond our initial goal of detecting IR excesses, we are interested in determining the properties of the dust in each system: its temperature, luminosity, mass, size distribution, composition, 
TABLE 4

Dust Emission at 24 and $70 \mu \mathrm{m}$ (IN mJy)

\begin{tabular}{|c|c|c|c|c|}
\hline Star & $F_{\nu}(24)_{\mathrm{dust}}$ & $F_{\nu}(70)_{\text {dust }}{ }^{\mathrm{a}}$ & $T_{\text {dust }}^{\mathrm{b}}$ & $L_{\text {dust }} / L_{*}{ }^{\mathrm{c}}$ \\
\hline HD $25998 .$. & $18.6 \pm 5.9$ & $51.1 \pm 9.6$ & $96 \pm 5$ & $4.5 \times 10^{-5}$ \\
\hline HD $38858^{\mathrm{d}}$. & $<15(3 \sigma)$ & $193 \pm 25$ & $<70$ & $12 \times 10^{-}$ \\
\hline HD $40136 \ldots$ & $63 \pm 22$ & $37.9 \pm 11$ & $165_{-20}^{+35}$ & $1.9 \times 10^{-5}$ \\
\hline HD $48682^{\mathrm{d}}$. & $<22(3 \sigma)$ & $290 \pm 38$ & $<68$ & $1.1 \times 10^{-4}$ \\
\hline HD $90089 \ldots$. & $<18(3 \sigma)$ & $23.2 \pm 5.0$ & $<120$ & $0.8 \times 10^{-5}$ \\
\hline HD $105211^{\mathrm{d}}$. & $<45(3 \sigma)$ & $521 \pm 73$ & $<70$ & $7.3 \times 10^{-5}$ \\
\hline HD $109085^{\mathrm{d}}$. & $292 \pm 24$ & $212 \pm 28$ & $150 \pm 10$ & $15 \times 10^{-5}$ \\
\hline HD $139664^{d} .$. & $<75(3 \sigma)$ & $523 \pm 77$ & $<78$ & $12 \times 10^{-5}$ \\
\hline HD $158633 \ldots \ldots \ldots . . . .$. & $<13(3 \sigma)$ & $45.8 \pm 7.8$ & $<90$ & $3.5 \times 10^{-5}$ \\
\hline HD $199260 \ldots \ldots \ldots \ldots . . . . .$. & $11.6 \pm 5.6$ & $32.9 \pm 6.4$ & $94 \pm 5$ & $3.3 \times 10^{-5}$ \\
\hline HD $219482 \ldots \ldots \ldots \ldots . . . .$. & $9.8 \pm 5.6$ & $54.5 \pm 9.0$ & $81 \pm 3$ & $3.6 \times 10^{-5}$ \\
\hline HD $219623 \ldots \ldots \ldots \ldots . . .$. & $<17(3 \sigma)$ & $34.8 \pm 6.5$ & $<104$ & $1.6 \times 10^{-5}$ \\
\hline
\end{tabular}

${ }^{\text {a }}$ Dust fluxes at $70 \mu \mathrm{m}$ have been color corrected by $8 \%$.

b Blackbody temperature based on either the $24 \mu \mathrm{m} / 70 \mu \mathrm{m}$ flux density ratio or the $70 \mu \mathrm{m}$ flux density plus a $3 \sigma$ upper limit at $24 \mu \mathrm{m}$.

${ }^{c}$ If only $70 \mu \mathrm{m}$ data are available, $L_{\text {dust }} / L_{*}$ is from eq. (3). If 24 and $70 \mu \mathrm{m}$ data are available, $L_{\text {dust }} / L_{*}$ is obtained from eq. (4).

${ }^{\mathrm{d}}$ For the resolved sources, the emission is fitted with an extended Gaussian profile, resulting in measured dust fluxes $\sim 10 \%$ higher than from the standard aperture photometry.

orbital location, etc. For the 12 stars with significant IR excess, Table 4 lists the excess $70 \mu \mathrm{m}$ emission and measurements of or limits to the $24 \mu \mathrm{m}$ excess. Seven of the 12 excess sources have an excess measured only at one wavelength $(70 \mu \mathrm{m})$, restricting our ability to place limits on key quantities. The observed flux can be translated into the total dust disk luminosity relative to its parent star only when some assumption is made for the dust temperature (e.g., Beichman et al. 2005a; Bryden et al. 2006). The minimum disk luminosity as a function of $70 \mu \mathrm{m}$ dust flux density is obtained by setting the emission peak at $70 \mu \mathrm{m}$ (or, equivalently, setting $T_{\text {dust }}=52.5 \mathrm{~K}$ ):

$$
\frac{L_{\text {dust }}}{L_{*}}(\text { minimum })=10^{-5}\left(\frac{5600 \mathrm{~K}}{T_{*}}\right)^{3} \frac{F_{\nu}(70 \mu \mathrm{m}, \text { dust })}{F_{\nu}(70 \mu \mathrm{m}, *)} .
$$

Based on this equation, a minimum $L_{\text {dust }} / L_{*}$ is calculated for each of our target stars identified as having an IR excess at only $70 \mu \mathrm{m}$ (Table 4). For the other IR-excess stars, we also have at least a rough $(2 \sigma)$ detection of the $24 \mu \mathrm{m}$ excess, as discussed in the previous section. With two wavelengths of excess measurement, the dust emission can be fitted with a representative dust temperature; otherwise, only upper limits can be obtained (Table 4; also see Fig. 13 below). Figure 8 shows a spectral energy distribution (SED) for two of these stars, HD 219482 and HD 40316, fitted with temperatures of 170 and $80 \mathrm{~K}$, respectively. In the cases with a fitted dust temperature, Table 4 lists the ratio of the integral under the stellar $\left(T_{*}\right)$ and dust $\left(T_{\text {dust }}\right)$ blackbodies as a proper, rather than minimum, estimate of $L_{\text {dust }} / L_{*}$ :

$$
\frac{L_{\text {dust }}}{L_{*}}=\left(\frac{T_{\text {dust }}}{T_{*}}\right)^{4}\left(\frac{e^{x_{\text {dust }}}-1}{e^{x_{*}}-1}\right) \frac{F_{\nu}(70 \mu \mathrm{m}, \text { dust })}{F_{\nu}(70 \mu \mathrm{m}, *)},
$$

where $x \equiv h \nu / k T=205.7 \mathrm{~K} / T$ at $70 \mu \mathrm{m}$.

For each of the stars with excess emission (plus those from Bryden et al. 2006), Figure 9 shows the total dust area and radial location of the emitting material. Despite the expectation that only large grains should be seen around mature systems due to loss mechanisms such as Poynting-Robertson drag and radiation pres-

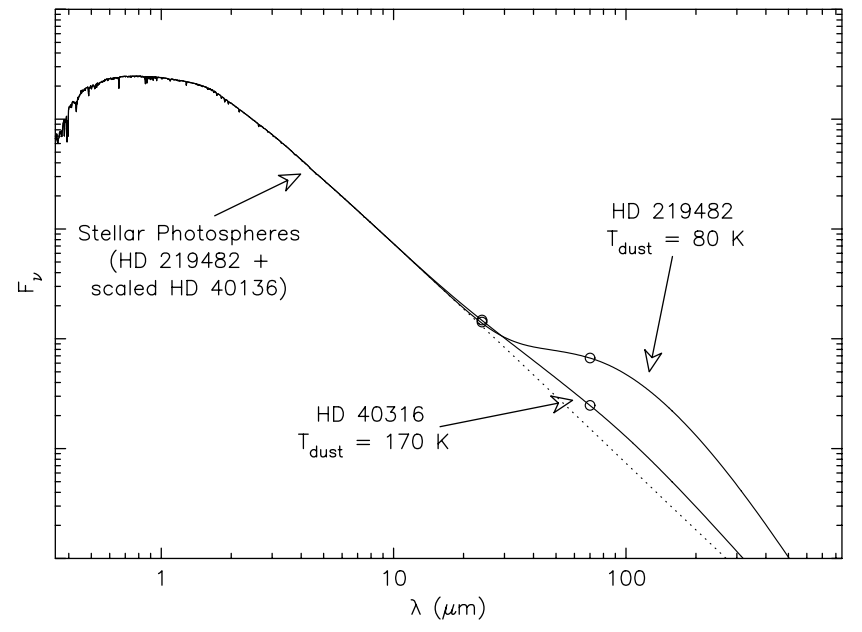

FIG. 8.-SEDs for two stars with IR excess at both 24 and $70 \mu \mathrm{m}$. The emission from the two stars has been scaled such that their photospheres overlap, emphasizing the difference in far-IR emission. The observed fluxes at each wavelength are shown as open circles that are fitted with a combination of emission from the stellar photosphere (dotted line) and from orbiting dust. The dust emission of HD 40316 is fitted with $170 \mathrm{~K}$ dust, whereas HD 219482, with stronger $70 \mu \mathrm{m}$ and weaker $24 \mu \mathrm{m}$ emission, is fitted with cooler, $80 \mathrm{~K}$ dust.

sure, small grains must be considered as a serious possibility given their presence in a mature star such as HD 69830 (Beichman et al. 2006) and because of the large extent of at least some of the dust disks. Thus, the orbital location of the emitting material can only be calculated if some assumption is made for the dust's emissivity; small grains are less efficient at emitting infrared radiation, resulting in a higher temperature for a given orbital radius. For a given dust temperature, the orbital radius decreases with emissivity as

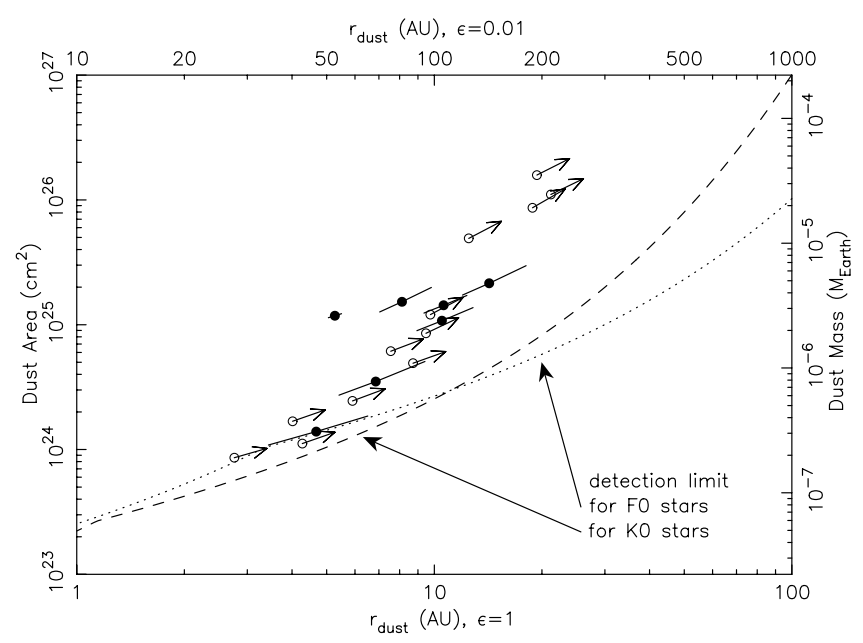

FIG. 9.-Area and dust mass estimates for stars with $70 \mu \mathrm{m}$ excess emission. In addition to our 12 stars with excess, the seven excess stars from Bryden et al. (2006) are also shown. Dust temperatures (Table 4) are translated into orbital radii assuming either large blackbody grains (bottom axis) or small grains with emissivity 0.01 (top axis). Stars with excess measured at both 24 and $70 \mu \mathrm{m}$ are shown as filled circles, while those with only upper limits for the dust temperature are shown as open circles. Error bars are added to each point based on the $1 \sigma$ uncertainties in the dust temperature; for systems with upper limits on the dust temperature, an arrow is plotted with length/direction based on an assumed $10 \%$ uncertainty in the temperature. Dust masses (right axis) are calculated assuming a typical grain size of $10 \mu \mathrm{m}$. Both dust area and mass are calculated under the assumption of blackbody grains (unity emissivity); for an emissivity of 0.01 , both area and mass are a factor of 100 larger. The detection limits, which depend on the stellar temperature, are shown for an F0 star (dotted line) and for a K0 star (dashed line). 
$\epsilon^{-0.5}$. As such, for each calculated dust temperature or upper limit in Table 4, two locations are plotted: the location if the emitting material is large blackbodies (lower axis), and the location if it is small grains with emissivity $=0.01$ (upper axis).

The dust area and mass have a similar ambiguity based on the unknown dust size/emission properties. The dust area in Figure 9 (left axis) is calculated under the assumption of blackbody grains (unity emissivity); a lower emissivity would increase the dust area in direct proportion to $\epsilon$. Finally, the dust mass (right axis) is based on an assumed typical grain size of $10 \mu \mathrm{m}$. An unconstrained amount of mass is contained in the larger parent bodies whose collisions produce the emitting dust.

For stars with no detected emission, $3 \sigma$ upper bounds on the $70 \mu \mathrm{m}$ fluxes lead to upper limits on $L_{\text {dust }} / L_{*}$ as low as a few times $10^{-6}$, assuming a dust temperature of $\sim 50 \mathrm{~K}$ (Table 2). Although we cannot rule out cold dust at $\gtrsim 100 \mathrm{AU}$, we are placing constraints on dust at Kuiper Belt distances to $\sim 10-100$ times the level of dust in our solar system. The constraint on asteroid belt-type dust is less stringent, $\sim 100-1000$ times our zodiacal emission.

\subsection{Comparison with Submillimeter Observations}

The dust properties can be further constrained with submillimeter flux measurements. When available, longer wavelength data can help place lower limits on dust temperature, upper limits on the dust luminosity, and, with some assumption on the grain emissivity, outer limits on the disk extent. For most of our stars with IR excess, large amounts of cold dust emitting at longer wavelengths cannot be ruled out, but three of the $70 \mu \mathrm{m}$ excess stars have been observed at 450 and $850 \mu \mathrm{m}$ with JCMT/SCUBA, with two detections (HD 48682 and HD 109085) and one upper limit (HD 139664; Sheret et al. 2004). Combining their submillimeter data with the infrared fluxes from IRAS, Sheret et al. (2004) modeled the SEDs for these stars, obtaining dust temperatures of 99 and $85 \mathrm{~K}$ for HD 48682 and HD 109085, respectively.

\subsection{Comparison with Visible Observations}

A nearly edge-on disk around HD 139664 has recently been detected at visible wavelengths using HST (Kalas et al. 2006). The HST image of the disk shows a well-defined inner edge around $83 \mathrm{AU}$ and an outer edge that extends out to about $109 \mathrm{AU}$. If the dust associated with the $70 \mu \mathrm{m}$ emission is located in this ring, then a very large surface area of $10 \mu \mathrm{m}$ grains would be required for the IR emission, since dust at this distance would have an equilibrium temperature of $30-35 \mathrm{~K}$ using a standard radial power law for grain temperature (Beichman et al. 2006). Smaller, $0.25 \mu \mathrm{m}$ grains, on the other hand, give temperatures of 69 and $77 \mathrm{~K}$ at the ring boundaries (cf. our $3 \sigma$ upper limit of $78 \mathrm{~K}$; Table 4). Using a simple relationship for dust mass, $M_{\text {dust }}=$ (4/3) $\rho a_{\text {grain }}\left[D 2 F_{\nu}\right.$ (dust) $\left./ Q_{\mathrm{abs}} B_{\nu}\left(T_{\text {dust }}\right)\right]$, and standard silicate absorption efficiencies, $Q_{\text {abs }}$ (Draine \& Lee 1984; Beichman et al. 2006), yields a mass of $2.4 \times 10^{-3} M_{\oplus}$ in large grains or $1.6 \times$ $10^{-4} M_{\oplus}$ in small grains, where we have taken a grain density of $\rho=3.3 \mathrm{~g} \mathrm{~cm}^{-3}$ and a distance of $D=17.5 \mathrm{pc}$ for this star.

The radiative blowout size for grains around an F5 star is approximately $1 \mu \mathrm{m}$ ( $\S$ V.B.1 in Backman \& Paresce 1993; Burns et al. 1979). In contrast to the spherical distribution of small grains seen toward Vega ( $\mathrm{Su}$ et al. 2005), which is probably due to a recent catastrophic event, small grains should be quickly ejected from the presumably stable ring system of HD 139664. IRS spectroscopy and/or millimeter spectroscopy would help distinguish between the large- and small-grain models.

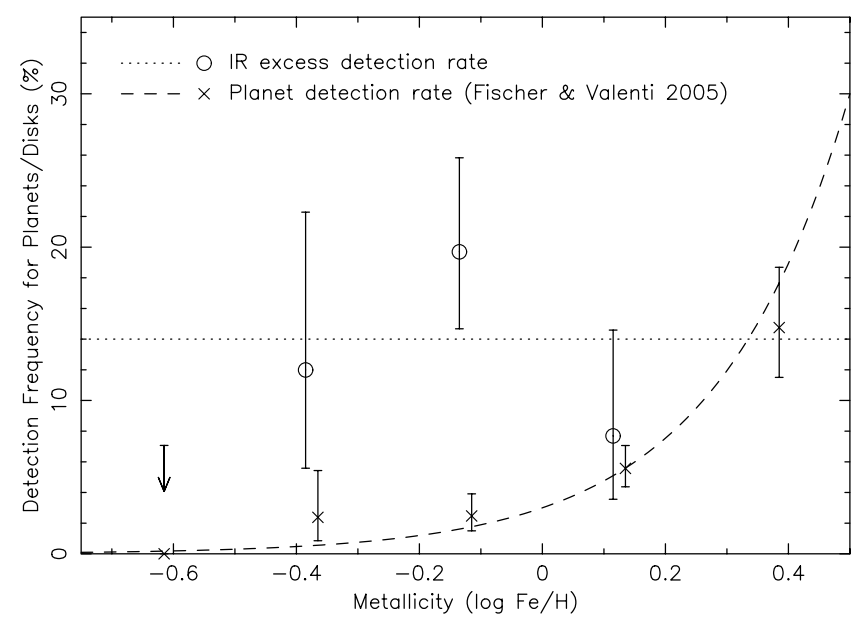

FIG. 10.-Detection frequency of planets and IR excess as a function of stellar metallicity. For the stars presented in this paper, plus those of Bryden et al. (2006), the detection rate of $70 \mu \mathrm{m}$ excess emission is shown as open circles. The distribution has no trend in metallicity; all points are consistent with the average detection rate for the entire sample (14\%; dotted line). This is in contrast to the dependence of the planet detection rate on metallicity for a similar sample of nearby stars (crosses), which Fischer \& Valenti (2005) fit with a metallicity squared relationship (dotted line).

\section{CORRELATION OF EXCESS WITH SYSTEM PARAMETERS}

To understand the origin and evolution of infrared excess, we now consider the properties of the sample stars and how they correlate with excess detection. Specifically, we examine the correlation with three variables: metallicity, age, and spectral type. These parameters are listed for each star in Table 1. Where appropriate we merge the present sample with that of Bryden et al. (2006) to improve the significance of any statistical conclusions.

\subsection{Metallicity}

Table 1 lists the metallicity information obtained from the literature for each of our target stars (number of independent $[\mathrm{Fe} / \mathrm{H}]$ estimates, their average, and their rms scatter). Figure 3 shows a histogram of these metallicity values, the majority of which range between -0.4 and +0.1 dex with a mean value of somewhat below solar. The stars with IR excess are identified with vertical arrows with the length of each arrow proportional to the strength of $70 \mu \mathrm{m}$ excess. We find no correlation between metallicity and IR excess. The average $[\mathrm{Fe} / \mathrm{H}]$ is $-0.15 \pm 0.03$ for all of the observed stars and $-0.17 \pm 0.04$ for the stars with excess, an insignificant difference.

These data are combined with the Bryden et al. (2006) sample to show the fractional incidence of disks as a function of $[\mathrm{Fe} / \mathrm{H}]$ (Fig. 10). A $\chi^{2}$ test shows that the distribution of disks in our three metallicity bins with a significant number of stars $(-0.75<$ $[\mathrm{Fe} / \mathrm{H}]<0.0)$ is indistinguishable from flat. The lack of correlation between IR excess and metallicity is in sharp contrast with the well-known correlation between extrasolar gas giant planets and host star metallicity (Gonzalez 1997; Santos et al. 2001). In particular, Fischer \& Valenti (2005) find that the probability of harboring a radial velocity-detected planet increases as the square of the metallicity (Fig. 10). Although one might suspect giant planets and debris disks to be related, a similarly strong correlation between dust and metallicity can be confidently ruled out. A $\chi^{2}$ comparison between the disk and planet distributions in the same three $[\mathrm{Fe} / \mathrm{H}]$ bins suggests that there is only a $0.3 \%$ probability of these being drawn from the same distribution. 


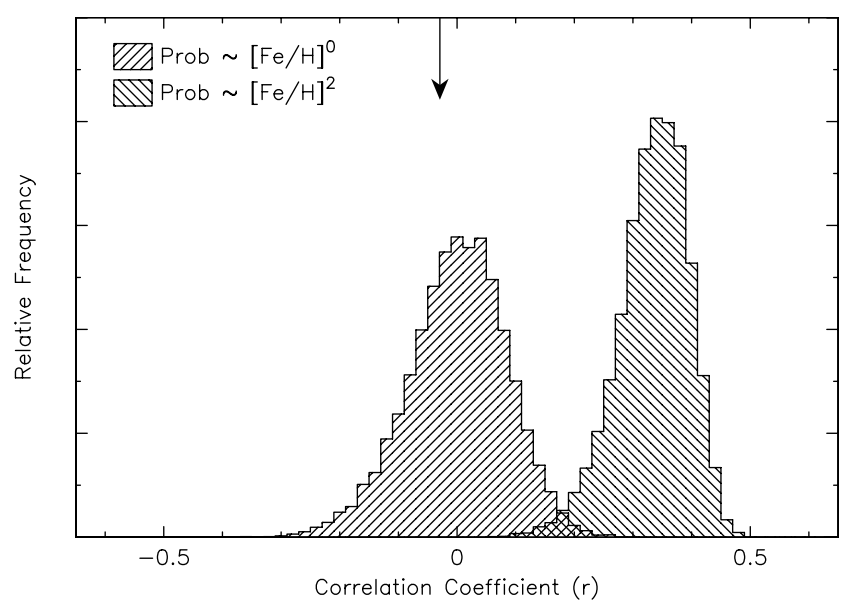

FIG. 11.-Distribution of $r$ correlation coefficients for two series of Monte Carlo simulations. Stars were selected either completely randomly (left histogram) or proportionate to their metallicity squared (right histogram), the relationship observed for planet-bearing stars. The arrow at the top shows the value of $r$ observed within our data, strongly inconsistent with the planet-metallicity relationship.

The lack of correlation is further confirmed via Monte Carlo simulations. Again using our data set combined with that of Bryden et al. (2006) (giving a total of 19 excess stars out of 151 observed), the correlation coefficient, $r$, is calculated for 10,000 random samples of stars. The histogram of the resultant $r$-values is shown in Figure 11 under two different assumptions. In one case, the stars with excess are chosen randomly (left histogram centered on $r=0$ ); in the other, the stars are chosen with weighting proportional to their metallicity squared (right histogram with average $r=0.33$ ). The correlation coefficient observed within our data (vertical arrow) is inconsistent with the strong metallicity dependence observed within planet-bearing stars.

This lack of correlation may reflect the different formation histories of giant planets and debris disks. The accretion of gas onto a giant planet requires a large solid core to form first, favoring a higher metallicity disk, whereas dust emission indicates the presence of smaller planetesimals that might be able to form in all disk environments. Another explanation may be that debris disks in high-metallicity systems initially contain more material, but that over time all disks grind down toward similar masses (e.g., Dominik \& Decin 2003). In this case, the detection of strong IR emission is a reflection of a recent stochastic collision, rather than the disk's initial conditions (for further discussion see Bryden et al. 2006).

\subsection{Age}

Collisions in a debris disk continually grind down the larger planetesimals, while the smallest dust can be removed by PoyntingRobertson drag and radiation pressure. One would assume that the overall disk mass must decline with time and, as expected, a correlation between stellar age and IR excess is observed, with debris disks more commonly identified around younger stars. While studies concentrating on stars younger than 1 Gyr find a strong trend (Spangler et al. 2001; Rieke et al. 2005), among nearby solar-type field stars the correlation is relatively weak (Bryden et al. 2006). In both cases, the evolution of the dust does not appear to be a steady decline. Observations of A stars find an overall decline in the average amount of $24 \mu \mathrm{m}$ excess emission on a $\sim 150$ Myr timescale, but the large variations on top of this trend suggest that sporadic collisional events are able to dramatically increase the amount of dust even at late stages in the disk's evolution (Rieke et al. 2005). As a result of these collisions, even old stars can have strong IR emission (Habing et al. 2001; Decin et al. 2000; Bryden et al. 2006).

Figure 2 shows the resultant histogram of stellar ages. The ages for our main-sequence stars are difficult to determine, with uncertainties in many cases of at least a factor of 2 . Where possible, we use ages based on $\mathrm{Ca}$ II $\mathrm{H}$ and $\mathrm{K}$ line emission from the large compilation of Wright et al. (2004). Otherwise, an average of values found in the literature is used. If the star was inferred to be young due to kinematic properties (Montes et al. 2001), we adopted that age. Table 1 lists the age data for each star. Although our target selection criteria do not explicitly discriminate based on stellar age, young stars (ages less than 1 Gyr) are not well represented in our sample due to their infrequent occurrence within $\sim 25$ pc of the Sun.

As in our earlier survey of nearby main-sequence stars (Bryden et al. 2006), the stars with excess in this survey (marked with arrows in Fig. 2) have a weak but noticeable correlation with stellar age. No stars older than 7 Gyr have a significant amount of excess emission. The average age of stars with IR excess is $4.0 \pm 0.6 \mathrm{Gyr}$, compared to $5.6 \pm 0.4$ Gyr for the sample as a whole. As discussed in the next section, these trends are present in the combination of this sample with the Bryden et al. (2006) data.

\subsection{Spectral Type}

Observations of the general characteristics of debris disks as a function of spectral type are potentially a powerful tool for understanding the physical mechanisms responsible for the evolution of debris disks. The disk properties should be directly related to the stellar mass and luminosity in several ways. The mass of the protostellar disk from which the debris formed, for example, probably depends on the parent star's mass, as does its dynamical timescale. The stellar luminosity, however, is undoubtedly more important for debris disk characteristics, exerting a strong influence on the typical particle size $\left(r_{\text {blowout }} \propto L_{*}\right)$, as well as its temperature $\left(T_{\text {dust }} \propto L_{*}^{0.2-0.25}\right)$. There are also observational biases linked to the brightness of the star, with cool dust seemingly easier to distinguish around hotter stars.

The minimum $L_{\mathrm{dust}} / L_{*}$ based on the $70 \mu \mathrm{m}$ flux, for example, is strongly dependent on stellar temperature (in eq. [3], detectable $L_{\text {dust }} / L_{*}$ is proportional to $\left.T_{*}^{-3}\right)$. Hotter stars emit a lower fraction of their luminosity at infrared wavelengths, allowing for better contrast at those wavelengths. But while equation (3) is an observationally well defined quantity, it contains no knowledge of the underlying disk physics. Naively, it appears to dictate a strong relationship between detectability and spectral type, i.e., it is easier to detect dust around hotter stars, but this may be misleading. For lack of any other information, the equation assumes that the dust emission peaks at $70 \mu \mathrm{m}$, thereby measuring the minimum $L_{\text {dust }} / L_{*}$. This assumed SED shape corresponds to a fixed dust temperature of $\sim 50 \mathrm{~K}$ for all disks. One can instead consider disk models with a more physically motivated dependence on spectral type. Instead of assuming a constant dust temperature, Habing et al. (2001), for example, assume the same dust location for all disks; in their models, the dust resides at $50 \mathrm{AU}$ independent of spectral type. In this case, dust temperature decreases with $T_{*}$. In contrast with a simple reading of equation (3), the Habing et al. (2001) models have $L_{\text {dust }} / L_{*}$ more or less directly proportional to $F_{\text {dust }} / F_{*}$ for stars of type $\mathrm{G}$ and earlier. As in equation (3), lower stellar temperature makes it more difficult to detect dust emission relative to the stellar photosphere, but in the Habing et al. (2001) models this difficulty is offset by a lower dust temperature for cooler stars, increasing the dust's $70 \mu \mathrm{m}$ emission. 


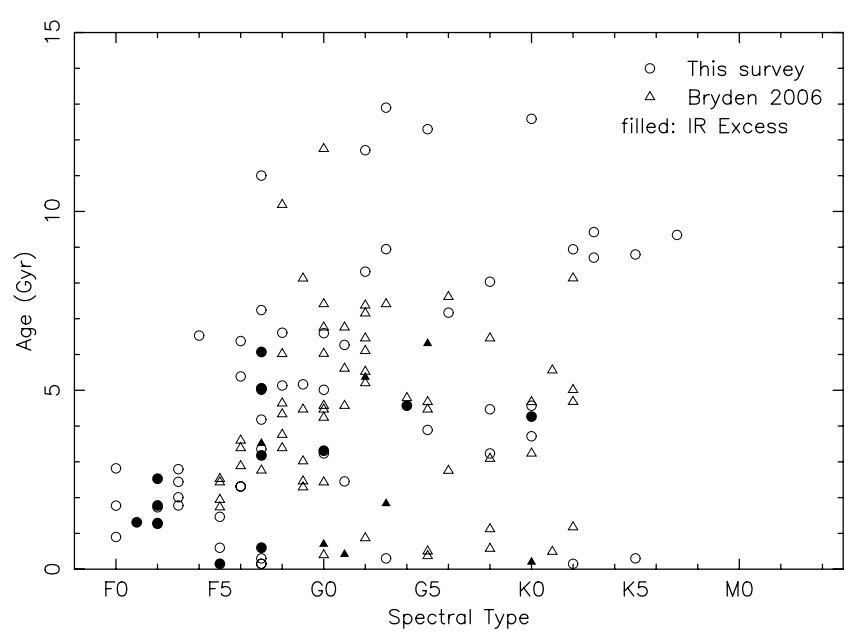

FIG. 12.- Stellar age as a function of spectral type for stars with known ages. The stars from this survey are marked with circles, while those from Bryden et al. (2006) are marked with triangles. In both cases, stars with IR excess are marked with filled symbols.

In Figure 1, the spectral types with IR-excess stars are flagged with vertical arrows. A clear trend is readily apparent, with excess more frequently detected around earlier type stars. The detection rate drops from nearly $30 \%$ for the earliest type stars down to $0 \%$ for $\mathrm{M}$ stars. In fact, no stars with spectral type later than $\mathrm{K} 0$ are found to have excess emission (a sample of 23 stars without excess). This is consistent with previous survey results that considered only part of the spectral range covered here. Our survey of F5-K5 stars (Bryden et al. 2006) found a detection rate of $13 \%$ within this limited spectral range, while a sample of $\sim 30$ images of nearby M stars yielded none with IR excess at $70 \mu \mathrm{m}$ (T. N. Gautier et al. 2006, in preparation).

A possible interpretation of the trend with spectral type is that it simply reflects the known correlation with stellar age. Earlier spectral type stars tend to be younger. Figure 12 combines information on spectral type and age into a single plot for stars in this survey and those of Bryden et al. (2006). The trends previously identified are apparent: an upper limit to the ages of stars with excesses ( filled symbols) of about 6 Gyr and a tendency for earlier type stars to have excess more frequently than later types. While the earliest type stars (F0-F3) are clearly younger on average, there is no clear evidence within the bulk of the sample that higher mass stars have more frequent excess because they have younger ages. The formal correlation of excess with spectral type is even stronger than the correlation with age (correlation coefficients are $-0.20 \pm 0.08$ and $-0.15 \pm 0.08$ for spectral type and age, respectively), further suggesting that spectral type is an independent indicator for IR excess. Unfortunately, many of the latest type stars lack reliable age indicators, making it difficult to make any stronger conclusions.

\subsubsection{Comparison with Early-Type Stars}

The detection rate of $70 \mu \mathrm{m}$ excess around A stars is $33 \% \pm$ $4 \%$ (Su et al. 2006), more than twice that for the stars considered in this paper $(13 \% \pm 3 \%)$. However, the A star and FGK star samples differ in both mass and age. We first consider the possibility that the different detection rates simply reflect an age evolution, rather than a spectral type dependence. For example, the youngest FGK stars have a detection rate somewhat higher than that within the sample as a whole: considering only systems with ages of 0.1-1 Gyr (and including the stars from Bryden et al. 2006), 5 out of 19 young FGK stars have excess $70 \mu \mathrm{m}$ emission $(=26 \% \pm$

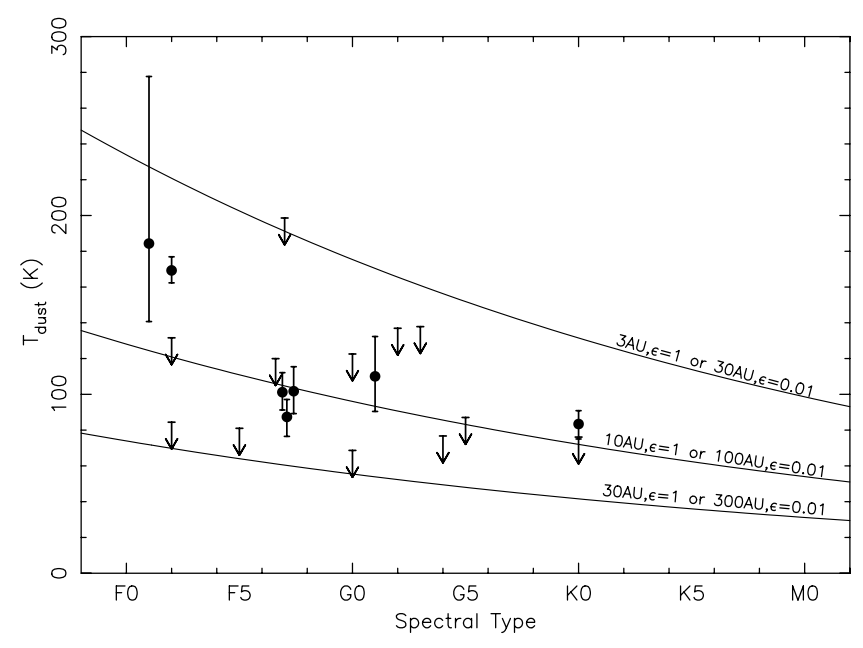

FIG. 13.-Dust temperature for stars with IR excess in this sample and from Bryden et al. (2006). For stars with excess measured at both 24 and $70 \mu \mathrm{m}$ ( filled circles), the dust SED is fitted with a representative temperature. Those stars with a single measurement of excess at $70 \mu$ m only have $(3 \sigma)$ upper limits. With some assumptions for the grain properties, the dust temperatures can be translated to orbital distances. Several lines of constant distance are shown for comparison. The observed systems with measured dust temperatures are mostly consistent with large blackbodies orbiting at $\sim 10 \mathrm{AU}$ or small, low-emissivity grains at $\sim 100 \mathrm{AU}$. There is no clear evidence for orbital distance changing as a function of spectral type.

$12 \%)$. Similarly, the $70 \mu \mathrm{m}$ excess frequency among the A stars drops with stellar age down to just $21 \% \pm 6 \%$ for A stars $0.3-1 \mathrm{Gyr}$ old (Su et al. 2006). It is important to note, however, that many of the $\mathrm{Su}$ et al. (2006) observations are less sensitive than those presented here, relative to the stellar photosphere. Thus, their A star detection rate should be regarded as a lower limit. Although the FGK and A star samples have stellar age as the most important correlating factor for IR excess, we cannot rule out a weaker but still important dependence of IR excess on some factor related to stellar mass such as luminosity or disk mass.

\subsubsection{Comparison with Late-Type Stars}

Combining the observations presented here with those of Bryden et al. (2006) and T. N. Gautier et al. (2006, in preparation), we have a total sample of 61 K1-M6 stars with no evidence of excess emission at $70 \mu \mathrm{m}$. Even considering only those stars whose photospheres are detected at $70 \mu \mathrm{m}$ with $\mathrm{S} / \mathrm{N}>3$ (42 of the 61 stars), this lack of excess detections is $>3 \sigma$, inconsistent with the $\sim 15 \%$ detection rate around F- and G-type stars. As implied by equation (3), the contrast of dust relative to photosphere is, however, poorer for cooler stars, which emit more of their energy in the infrared than hotter stars. The average upper limit to $L_{\text {dust }} / L_{*}$ for the 16 stars K1 or later with detected photospheres but no excesses in the SIM PlanetQuest/TPF sample $\left[\mathrm{S} / \mathrm{N}(70 \mu \mathrm{m})>3\right.$ and $\left.\chi_{70}<3\right]$ is $L_{\text {dust }} / L_{*}<9 \times 10^{-6}$, compared with the average upper limit for 51 hotter stars with detected photospheres but no excesses, $L_{\text {dust }} / L_{*}<4 \times 10^{-6}$. Thus, one explanation for the lack of excesses around later type stars is simply that the effective observational limits are a factor of 2 higher for the cooler stars. While observational selection effects make detection of IR excess around late-type stars more difficult, the strength of this trend suggests that other explanations are needed.

Another ambiguity in interpreting the correlation of excess with spectral type results from our limited knowledge of the location of the dust. If dust around later type stars is very distant from its central star, it will be too cool for detection at $70 \mu \mathrm{m}$. Figure 13 shows how the dust temperature varies as a function of 


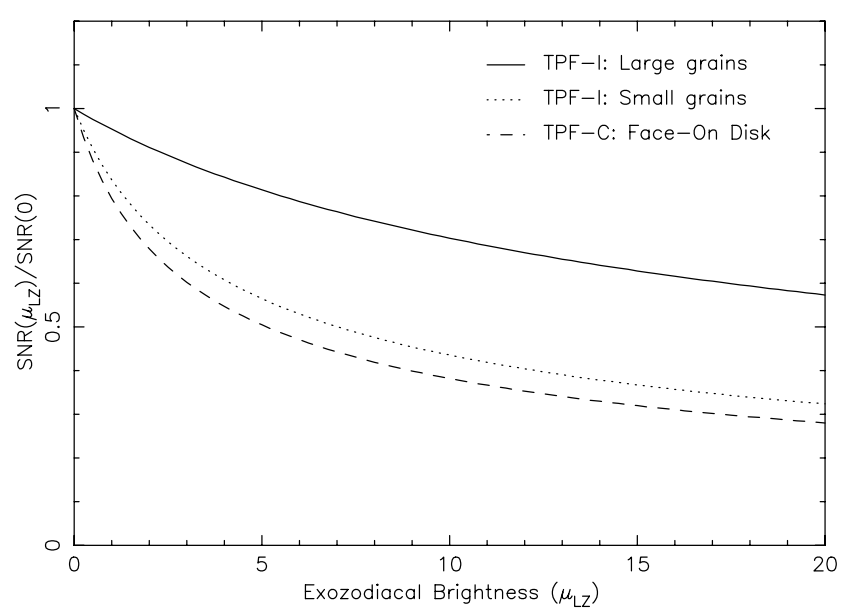

FIG. 14.-Effect of exozodiacal dust emission on TPF S/N. The horizontal axis gives the vertical optical depth of the exozodiacal disk normalized to that of the solar system $\left(\mu_{\mathrm{LZ}}\right)$. Note that a value of $\mu_{\mathrm{EZ}}=1$ in a target system corresponds to twice the emission we see from our zodiacal cloud, e.g., using $C O B E$ or IRAS, since we view our cloud from its midplane. The solid and dotted lines show the falloff in relative $\mathrm{S} / \mathrm{N}$ for the interferometer as the amount of exozodiacal emission increases, with large and small grain sizes considered separately (solid and dotted lines, respectively). The dashed line shows a similar trend for a coronagraph viewing a face-on disk. In each case, the $\mathrm{S} / \mathrm{N}$ is shown relative to observations of a system with no dust emission.

spectral type for stars with excess from both this sample and Bryden et al. (2006). We can only derive a dust temperature in the limited number of cases where we have a measured excess at both 24 and $70 \mu \mathrm{m}$ (filled circles). Otherwise, only upper limits can be obtained (Table 4). For unresolved disk observations, the dust location cannot be determined without some knowledge of the underlying dust emission properties. Smaller dust with low emissivity can be just as hot as larger grains closer to the central star. Lines of constant orbital radius are shown in Figure 13 under the assumption of either large blackbody grains $(\epsilon=1)$ or small grains with emissivity $=0.01$. Although the observed temperatures range from 80 to $170 \mathrm{~K}$, they are all more or less consistent with emission from similar orbital locations: $10 \mathrm{AU}$ for $\epsilon=1$ or $100 \mathrm{AU}$ for $\epsilon=0.01$. By implication, one would expect that dust around later $\mathrm{K}$ stars might have typical temperatures of $\sim 50 \mathrm{~K}$, ideal for detection at $70 \mu \mathrm{m}$, although none were detected.

Additional information on the location of the dust comes from IRS observations of $\mathrm{F}, \mathrm{G}$, and $\mathrm{K}$ stars with excesses (Beichman et al. 2006), which reveal that in almost all cases the inner boundary of the emitting region occurs at or interior to $10 \mathrm{AU}$. A theoretical basis for this preferred location of a radial distance of a few AU comes from the suggestion that the water ice sublimation distance, or the "snowline" where the temperature falls below $170 \mathrm{~K}$, should mark the onset of the region of giant planet formation and its remnants in the Kuiper Belt (Hayashi 1981; Sasselov \& Lecar 2000; Garaud \& Lin 2006). Since the location of the snowline varies with stellar luminosity $\left(\propto L^{0.25}\right)$, there is no reason to expect a more distant, hidden reservoir of material unsampled by our observations around cooler stars. It is, of course, important to verify this expectation with observations at longer wavelengths such as MIPS $160 \mu \mathrm{m}$ and in the submillimeter. Within the T. N. Gautier et al. (2006, in preparation) sample, for example, none of the $20 \mathrm{M}$ stars examined at $160 \mu \mathrm{m}$ show any excess emission, providing limits on $L_{\text {dust }} / L_{*}$ of $10^{-5}$ to $10^{-3}$ for material at $\sim 50 \mathrm{AU}$.

If the lack of debris disks around cool stars is real, then the dearth of material might reflect different formation mechanisms and evolutional history for the belts of planetesimals around low-
TABLE 5

Predicted Dust Emission at $10 \mu \mathrm{m}$

\begin{tabular}{|c|c|c|}
\hline Star & $\begin{array}{c}F_{\nu} \\
(\mathrm{mJy})\end{array}$ & $\begin{array}{c}\text { Exozodiacal }^{\mathrm{a}} \\
(\text { Solar System }=1)\end{array}$ \\
\hline HD 25998 & 0.041 & 1.7 \\
\hline HD 40136 & 5.1 & 42 \\
\hline HD 109085 & 23 & 325 \\
\hline HD 199260 & 0.024 & 1.1 \\
\hline HD 219482 & 0.004 & 0.2 \\
\hline
\end{tabular}

${ }^{\mathrm{a}} L_{\mathrm{dust}} / L_{*}$ at $10 \mu \mathrm{m}$ in units of $10^{-7}$, corresponding roughly to that of the solar system (Backman \& Paresce 1993). See eq. (2) of Beichman et al. (2006).

mass stars. Dust-producing collisions within these belts, for example, may require planetesimal stirring by larger, gas giant planets, whose frequency is thought to be lower for late-type stars (Laughlin et al. 2004), Alternately, the lack of IR excess might instead indicate a change in the physics of the smallest orbiting bodies as later type stars are considered, such as the increased relative importance of stellar winds in clearing dust from the system (Plavchan et al. 2005).

\section{APPLICABILITY TO TPF}

The detection of other terrestrial planets is a long-term goal for the astronomical community (McKee \& Taylor 2001). NASA has spent considerable funds over the past decade on technology development and mission studies for a TPF. One of the key astroengineering issues revealed by those studies is the level of dust emission associated with target stars since exozodiacal emission is potentially an important source of photon shot noise (Beichman et al. 1999). Thus, in addition to scientific interest, the incidence and distribution of material in the habitable zones, i.e., where planets might have surface temperatures consistent with the presence of liquid water (Kasting et al. 1993), of nearby stars are of considerable technical importance.

\subsection{Effect of Exozodiacal Dust on Planet Finding}

As discussed in the Appendix, dust emission at the level of 10-20 times that of our own zodiacal cloud can impede planet searches (Fig. 14) due to increased photon shot noise for either a coronagraph or an interferometer. Since this level is roughly 50100 times less than that currently detectable with Spitzer, we can rule out only those stars with the most extreme zodiacal disks. Thus, HD 109085 and HD 69830 (Beichman et al. 2005b) are unsuitable targets with strong excess shortward of $24 \mu \mathrm{m}$. However, the remaining stars in this sample and other samples pass the initial screening by having $24 \mu \mathrm{m}$ excesses, if any, less than $L_{\text {dust }} / L_{*} \simeq 10^{-4}$, corresponding to upper limits on $\mu_{\mathrm{EZ}} \sim 500$ (Bryden et al. 2006). Beyond these photometric constraints, IRS spectroscopy can push upper limits to factors of 2-3 lower than MIPS alone and can also identify stars with small-grain emission at $10 \mu \mathrm{m}$ (Beichman et al. 2006).

In a few cases listed in Table 5 we can use the blackbodies fitted to the emission from the five stars with data at both 24 and $70 \mu \mathrm{m}$ (Table 4) to extrapolate the emission from this "Kuiper Belt" dust to the prime TPF- $I$ wavelength of $10 \mu \mathrm{m}$. The extrapolated emission is also given in units of $L_{\text {dust }} / L_{*}$ for material emitting at $10 \mu \mathrm{m}$ (see eq. [3] and eq. [2] of Beichman et al. 2006) relative to the solar system value of $10^{-7}$ (Backman \& Paresce 1993). Emission from any of this material located within the primary beam of the TPF-I telescopes $(r<5$ AU for a star at 10 pc observed with $3 \mathrm{~m}$ apertures) would be a noise source as 
described in the Appendix. However, this population of "cool" or "lukewarm" grains would not be located within a TPF-C pixel centered on the $\sim 1$ AU habitable zone and would not be a noise source at visible wavelengths.

Unfortunately, however, the present observations cannot rule out an additional population of hotter grains located closer to the star that would either emit at $10 \mu \mathrm{m}$ or scatter in the visible. IRS observations in the $8-14 \mu \mathrm{m}$ region reach levels of just 1000 times the zodiacal level (Beichman et al. 2006). It will take observations with nulling interferometers such as the Keck and Large Binocular Telescope Interferometers that can spatially suppress the stellar component to measure directly the exozodiacal emission in the habitable zone at levels that could cause $\mathrm{S} / \mathrm{N}$ or confusion problems for $T P F$.

There is some cause for optimism, however. The "luminosity function" of disks inferred from a variety of Spitzer samples (Bryden et al. 2006), the rarity of extreme "hot" zodiacal disks in the sample reported here and in other Spitzer papers (Bryden et al. 2006; Beichman et al. 2006), and the apparent decline in the number of stars with excesses as a function of age (Fig. 2) are all encouraging signs that the relatively clean example of our solar system may be the norm rather than the exception. The ringlike structures seen in a number of resolved Spitzer disks, e.g., Fomalhaut (Stapelfeldt et al. 2004) and $\epsilon$ Eri (D. E. Backman et al. 2006, in preparation), as well as in HST images (Kalas et al. 2005, 2006), suggest that although the regions interior to the rings may not be completely empty due to a variety of mechanisms capable of transporting material inward from the outer disk (comets, Poynting-Robertson drag, interactions with planets, etc.; Holmes et al. 2002), these interior regions may have a quite low total optical depth, perhaps as low as the $\sim 20 \%$ contribution inferred for material from Kuiper Belt material to the total amount seen at 1 AU in our solar system (Landgraf et al. 2002; Dermott \& Kehoe 2004; Moro-Martín \& Malhotra 2005).

\section{SUMMARY}

We have searched for circumstellar dust around a sample of $88 \mathrm{~F}-\mathrm{M}$ stars, by means of photometric measurements at 24 and $70 \mu \mathrm{m}$. We detected all of the stars at $24 \mu \mathrm{m}$ with high $\mathrm{S} / \mathrm{N}$ and more than $80 \%$ of the stars at $70 \mu \mathrm{m}$ with $\mathrm{S} / \mathrm{N}>3$. Uncertainties in the Spitzer calibration and in the extrapolation of stellar photospheres to far-IR wavelengths limit our ability to detect IR excesses with $3 \sigma$ confidence to $\sim 20 \%$ and $\sim 50 \%$ of the photospheric levels at 24 and $70 \mu \mathrm{m}$, respectively.

At these levels we have detected 12 of 88 objects with significant $70 \mu \mathrm{m}$ excesses. Combined with an earlier study (Bryden et al. 2006), we find an overall detection rate of $13 \% \pm 3 \%$ for mature cool stars. Beyond the single previously known $24 \mu \mathrm{m}$ excess within our sample, we detect two objects with $70 \mu \mathrm{m}$ excesses and definite but weak $24 \mu \mathrm{m}$ emission. Another two stars with $70 \mu \mathrm{m}$ excesses have $2 \sigma$ hints of $24 \mu \mathrm{m}$ excesses. These results build on the finding of Beichman et al. (2006) that, in many cases, objects with $70 \mu \mathrm{m}$ emission also had IRS spectra rising longward of $25 \mu \mathrm{m}$ to meet the $70 \mu \mathrm{m}$ excess. These objects are all consistent with a disk architecture similar to our Kuiper Belt that is concentrated outside 5-10 AU. In this context we note that a number of the $70 \mu \mathrm{m}$ sources are slightly, but significantly, extended at $70 \mu \mathrm{m}$. The detailed discussion of these objects is deferred to a subsequent paper (G. Bryden et al. 2006, in preparation). The IR emission in these systems is different from the exceptional object HD 69830, which shows a disk architecture much more consistent with a massive asteroid belt (Beichman et al. 2005b).

Cross-correlating the detections of IR excess with stellar parameters, we find no significant correlation in the incidence of excess with metallicity, but we do find weak correlations with both stellar age and spectral type. The lack of correlation with metallicity contrasts with the known correlation between planet detections and stellar metallicity and the expectation that higher metal content might result in a greater number of dust-producing planetesimals.

One significant finding is that the incidence of debris disks among mature stars is markedly lower for spectral types later than $\mathrm{K} 0$ than for earlier spectral types. Combining data from this survey, the Bryden et al. (2006) F5-K5 survey, and the T. N. Gautier et al. (2006, in preparation) M star survey suggests an incidence of disks of $15 \% \pm 3 \%$ for $\mathrm{F} 0-\mathrm{K} 0$ stars and $0 \% \pm 4 \%$ for stars with types K2-M3. This lack of disks around later spectral types may be due to selection effects, lower initial disk mass, or different rates of dust creation or destruction.

The disks that we are detecting have typical $70 \mu \mathrm{m}$ luminosities around 100 times that of the Kuiper Belt. If they also have inner asteroid belts 100 times brighter than our own, however, we would still not be able to detect this warm inner dust. The observed $70 \mu \mathrm{m}$ excess systems could all be scaled-up replicas of the solar system's dust disk architecture, differing only in overall magnitude. These systems could have planets, asteroids, and Kuiper Belt objects as in our own system, but simply with a temporarily greater amount of dust due to a recent collisional event. Further observations of the warmer inner dust are necessary to address this possibility. Spitzer IRS is particularly promising in this regard (Beichman et al. 2006) and is being pursued as part of a follow-up effort for some of the stars in this program.

This publication makes use of data products from the Two Micron All Sky Survey (2MASS), as well as from IPAC, SIMBAD, VIZIER, and the ROE Debris Disks Database Web site. We gratefully acknowledge the assistance of John Carpenter in reducing the IRAC data reported in this paper, and we thank Angelle Tanner and Kate Su for helpful discussions. The Spitzer Space Telescope is operated by the Jet Propulsion Laboratory, California Institute of Technology, under NASA contract 1407. Development of MIPS was funded by NASA through the Jet Propulsion Laboratory, subcontract 960785 . Some of the research described in this publication was carried out at the Jet Propulsion Laboratory, California Institute of Technology, under a contract with the National Aeronautics and Space Administration.

\section{APPENDIX}

\section{NOISE DUE TO EXOZODIACAL EMISSION}

In this section we make order-of-magnitude estimates of the impact of photon noise from exozodiacal emission on both visiblelight and mid-IR instruments (TPF-C and $T P F-I$, respectively) designed to find neighboring planets. A detailed noise analysis of planet-finding telescopes is beyond the scope of this paper, and the reader is referred to other articles for more details (Beichman $\&$ Velusamy 1999; Brown 2005). 


\section{A1. TPF-I, THE INFRARED INTERFEROMETER}

The use of a nulling interferometer to reject starlight and thereby reveal an orbiting planet dates to an article by Bracewell (1978) and has been further investigated through studies of more sophisticated configurations (Angel \& Woolf 1997; Lay et al. 2005). For a cryogenic system operating in an orbit near $1 \mathrm{AU}$, the three dominant noise sources are as follows (Beichman \& Velusamy 1999; Table 6): the stellar light that leaks past the interferometric null because of the finite diameter of the star, $S_{* \text {,leak }}$; emission from the local zodiacal dust, $S_{\mathrm{LZ}}$; and emission from the exozodiacal dust in the target star system that leaks past the interferometer, $S_{\mathrm{EZ} \text {, leak }}$ (see Fig. 15). At short wavelengths $(<8 \mu \mathrm{m})$, the stellar leak may dominate all other noise sources; longward of $20 \mu \mathrm{m}$, emission from a $35 \mathrm{~K}$ telescope will become important; and at all wavelengths various systematic instrumental effects will be important. But over a broad range of wavelengths, the balance between $S_{* \text {, leak }}, S_{\mathrm{LZ}}$, and $S_{\mathrm{EZ}, \text { leak }}$ controls the fundamental noise floor. Detector read noise and dark current can be ignored for broadband detection.

In the background limit considered here, the total noise is given by the square root of the sum of all of the individual photon fluxes reaching the detector. Rather than evaluate the absolute $\mathrm{S} / \mathrm{N}$, we consider here the ratio of the $\mathrm{S} / \mathrm{N}$ in the presence of exozodiacal emission, $\mathrm{S} / \mathrm{N}(\mathrm{EZ})$, to the $\mathrm{S} / \mathrm{N}$ in the absence of such emission, $\mathrm{S} / \mathrm{N}(0)$ :

$$
\left.\frac{\mathrm{S} / \mathrm{N}(\mathrm{EZ})}{\mathrm{S} / \mathrm{N}(0)}\right|_{\mathrm{IR}}=\frac{\sqrt{S_{*, \text { leak }}+S_{\mathrm{LZ}}}}{\sqrt{S_{*, \text { leak }}+S_{\mathrm{LZ}}+S_{\mathrm{EZ}, \text { leak }}}}=\frac{\sqrt{1+S_{*, \text { leak }} / S_{\mathrm{LZ}}}}{\sqrt{1+S_{*, \text { leak }} / S_{\mathrm{LZ}}+S_{\mathrm{EZ}, \text { leak }} / S_{\mathrm{LZ}}}} .
$$

In the above, $S_{* \text {, leak }}$ depends on the nulling configuration, the wavelength of operation, and the angular size of the star. Null depths of $10^{-5}$ to $10^{-6}$ have been demonstrated in the laboratory (Martin et al. 2003), and for the purposes of this illustration, it suffices to take $S_{* \text { leak }}=10^{-5} F_{*}$. The emission from the local zodiacal cloud, $S_{\mathrm{LZ}}$, is very complex in detail (Kelsall et al. 1998) but can be parameterized for our purposes as follows: $S_{\mathrm{LZ}}=\tau_{\mathrm{LZ}} B_{\nu}(255 \mathrm{~K}) \Omega_{\mathrm{tel}}$, where $B_{\nu}$ is the Planck function, $\tau_{\mathrm{LZ}}$ is the vertical optical depth looking out from the midecliptic plane at $1 \mathrm{AU}$, and $\Omega_{\text {tel }}$ is the diffraction-limited solid angle of an individual telescope in the interferometer. A typical value of the zodiacal cloud brightness toward the ecliptic pole from our midplane location is $12 \mathrm{MJy} \mathrm{sr}^{-1}$ at $12 \mu \mathrm{m}$ (Kelsall et al. 1998).

In the absence of more detailed information, the vertical optical depth of the exozodiacal dust in any system can be parameterized as a factor, $\mu_{\mathrm{EZ}}$, times the solar system's zodiacal dust. The emission from exozodiacal dust is then $S_{\mathrm{EZ}}(r)=2 \mu_{\mathrm{EZ}} \tau_{\mathrm{LZ}}(r) B_{\nu}[T(r)] \Omega_{\mathrm{tel}}$, where the factor of 2 accounts for the fact that in the exozodiacal case we are looking through the entire cloud and not from the vantage of the midplane as we do with the local cloud. By analogy with the local zodiacal cloud (Backman 1998), the vertical optical depth is assumed to fall off radially as $\tau_{\mathrm{LZ}}(r)=\tau_{\mathrm{LZ}, 1 \mathrm{AU}} r_{\mathrm{AU}}^{-0.3}$. We also take $T(r)=T_{0} r_{\mathrm{AU}}^{\beta}$ as the equilibrium temperature for grains heated by stellar radiation and emitting in the infrared. Typical 1 AU values of $\left(T_{0}, \beta\right)$ for large and small silicate grains are $(255 \mathrm{~K},-0.5)$ and (362 K, -0.4), respectively (Draine \& Lee 1984; Backman \& Paresce 1993; Beichman et al. 2006). The large- and small-grain brightness distributions are normalized to yield the same value at $1 \mathrm{AU}$.

The effect of exozodiacal emission is modulated by the fringe pattern of the interferometer, which attenuates the bright central portion of the exozodiacal disk. To account for this effect, we incorporate the fringe pattern of a particular nulling scheme $\zeta(\theta, \phi)$, where $\theta$ and $\phi$ are the radial and azimuthal variables, respectively. In the simplified case of a face-on disk, the signal reaching the detector, $S_{\mathrm{EZ}, \text { leak }}$, is then given by the integral of $S_{\mathrm{EZ}}$ over the fringe pattern and the telescope solid angle:

$$
S_{\mathrm{EZ}, \text { leak }}(d)=\int_{0}^{2 \pi} d \phi \int_{0}^{\theta_{\max }} \mu_{\mathrm{EZ}} \tau_{\mathrm{LZ}}(\theta d) B_{\nu}[T(\theta d)] \zeta(\theta, \phi) \theta d \theta,
$$

for a star at a distance $d$. We adopt the fringe pattern $\zeta(\theta, \phi)$ for the Dual Chopped Bracewell interferometer (DCB; Lay 2004; Lay et al. 2005) currently under study. Canceling out common factors, the stellar leak term in equation (A1) then becomes

$$
\left.\frac{S_{\mathrm{EZ}, \text { leak }}(d)}{S_{\mathrm{LZ}}}\right|_{\mathrm{IR}}=2 \mu_{\mathrm{EZ}}\left(e^{14,388 / \lambda T_{\mathrm{LZ}}}-1\right) \frac{1}{\Omega_{\mathrm{tel}}} \int_{0}^{2 \pi} d \phi \int_{0}^{\theta_{\max }} \frac{\zeta(\theta, \phi)(\theta d)^{-0.3}}{e^{14388 /[\lambda T(\theta d)]}-1} \theta d \theta .
$$

\begin{tabular}{|c|c|c|}
\hline Parameter & $T P F-C$ & $T P F-I$ \\
\hline Wavelength... & $0.55 \mu \mathrm{m}$ & $12 \mu \mathrm{m}$ \\
\hline Telescope & $3.5 \times 8 \mathrm{~m}$ & Four, $3 \mathrm{~m}$ on $75 \mathrm{~m}$ baseline \\
\hline 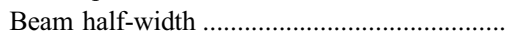 & $39 \times 17$ mas & 500 mas \\
\hline 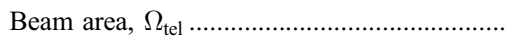 & $5 \times 10^{-14}$ & $1.8 \times 10^{-11}$ \\
\hline Local zodiacal emission, $I_{\mathrm{LZ}} \ldots \ldots \ldots \ldots \ldots \ldots \ldots \ldots$ & $0.1 \mathrm{MJy} \mathrm{sr}^{-1}$ & $12 \mathrm{MJy} \mathrm{sr}^{-1}$ \\
\hline Zodiacal flux density $\left(I_{\mathrm{LZ}} \Omega_{\text {tel }}\right) \ldots \ldots \ldots \ldots \ldots \ldots \ldots \ldots$ & $5 \mathrm{nJy}$ & $220 \mu \mathrm{Jy}$ \\
\hline Stellar magnitude ${ }^{a}$ & $V=4.5 \mathrm{mag}(60 \mathrm{Jy})$ & {$[12]=3.0 \mathrm{mag}(1.7 \mathrm{Jy})$} \\
\hline 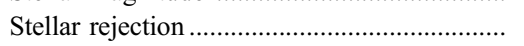 & $10^{-10}$ & $10^{-5}$ \\
\hline 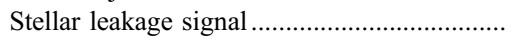 & $6 \mathrm{nJy}$ & $17 \mu \mathrm{Jy}$ \\
\hline 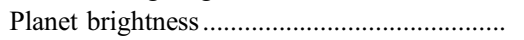 & $6 \mathrm{nJy}$ & $0.3 \mu \mathrm{Jy}$ \\
\hline
\end{tabular}

TABLE 6

Parameters For TPF S/N Calculations

${ }^{\mathrm{a}}$ For a solar twin at $10 \mathrm{pc}$. 


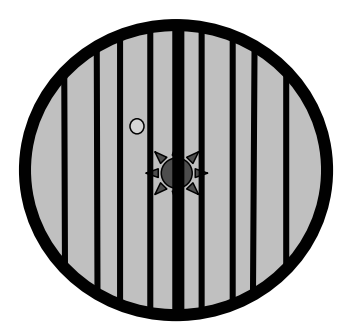

Infrared Interferometer

$r=0.5$ " beam or

$5 \mathrm{AU}$ at $10 \mathrm{pc}$

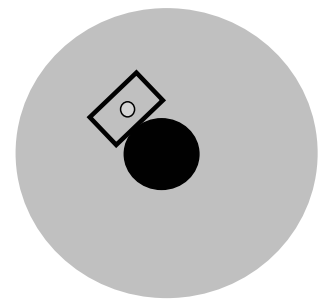

Visible Coronagraph

0.039 "x0.017" beam or

$0.4 \times 0.17 \mathrm{AU}$ at $10 \mathrm{pc}$

FIG. 15.- The interferometer (left) takes in all emission from a face-on exozodiacal disk that fits within the diffraction-limited beam of a single telescope. The intensity is, however, attenuated by the interferometric fringe pattern shown as vertical black bars. The coronagraph (right) takes in exozodiacal light only within the area of a single diffraction-limited pixel; the values shown here are appropriate for a $3.5 \times 8 \mathrm{~m}$ telescope under consideration for $T P F-C$.

To evaluate equation (A3), we adopt a diffraction-limited beam size of $\theta_{\max }=0.6 \lambda / D=0.5$ for a $D=3 \mathrm{~m}$ telescope at $12 \mu \mathrm{m}$. For a solar-type star at $d=10 \mathrm{pc}$, the ratio of the exozodiacal contribution to that from the solar system's own dust (eq. [A3]) is $0.06 \mu_{\mathrm{EZ}}$ and $0.24 \mu_{\mathrm{EZ}}$ for large and small grains, respectively. Warmer, smaller grains fill more of the beam of the individual telescopes than the cooler, larger (blackbody) grains and thus contribute more noise. With this information in hand, Figure 14 shows the variation of $\mathrm{S} / \mathrm{N}$ as a function of exozodiacal brightness, $\mu_{\mathrm{EZ}}$, for two grain sizes. When the exozodiacal surface density $\mu_{\mathrm{EZ}}$ is 10 times that of our solar system, corresponding to a 20 -fold brightness increase, the $\mathrm{S} / \mathrm{N}$ is reduced by a factor of $\sim 2$, necessitating an increase in integration time by a factor of $\sim 4$ to recover the original $\mathrm{S} / \mathrm{N}$. It is interesting to note the importance of grain size on this effect; the emission from the large grains is more centrally peaked and thus more effectively attenuated by the nulling interferometer than for the smaller grains, which remain warm at quite large distances from the star. Since at least a few hours of integration time are needed to detect an Earth in the presence of a $\mu_{\mathrm{EZ}}=1$ cloud, and days to carry out a spectroscopic program (Beichman 1998; Lay et al. 2005), it is clear that studying systems with $\mu_{\mathrm{EZ}}>10-20$ will be difficult.

\section{A2. $T P F-C$, THE VISIBLE-LIGHT CORONAGRAPH}

A similar analysis can be applied to an assessment of the effects of exozodiacal emission at visible wavelengths. There are some important differences, however. First, the coronagraph takes in only the exozodiacal light from the immediate vicinity of the planet, not from the entire exozodiacal cloud (Fig. 15, right panel). Second, the signal from an Earth $\left(S_{p}\right)$, the residual starlight after a $10^{-10}$ rejection ratio, and the local and exozodiacal signals are all more evenly balanced. Detector noise becomes a serious issue at medium spectral resolution $(\sim 75)$ but can be ignored in the broadband case. The analog of equation (A1) for the coronagraph becomes

$$
\left.\frac{\mathrm{S} / \mathrm{N}(\mathrm{EZ})}{\mathrm{S} / \mathrm{N}(0)}\right|_{\mathrm{Vis}}=\frac{\sqrt{1+S_{*, \text { residual }} / S_{\mathrm{LZ}}+S_{p} / S_{\mathrm{LZ}}}}{\sqrt{1+S_{*, \text { residual }} / S_{\mathrm{LZ}}+S_{p} / S_{\mathrm{LZ}}+S_{\mathrm{EZ}} / S_{\mathrm{LZ}}}}
$$

Since the local and exozodiacal emission enters the system through exactly the same solid angle, $\Omega_{\mathrm{tel}}$, the $S_{\mathrm{EZ}} / S_{\mathrm{LZ}}$ term simplifies to $2 \mu_{\mathrm{EZ}}$. For a planet $25 \mathrm{mag}$ fainter than a $V=4.5 \mathrm{mag}$ solar twin at $10 \mathrm{pc}$, and assuming a local zodiacal brightness of $0.1 \mathrm{MJy} \mathrm{sr}^{-1}$ at $0.55 \mu \mathrm{m}$ (Bernstein et al. 2002; Table 6), we can evaluate the variation in $\mathrm{S} / \mathrm{N}$ as a function of $\mu_{\mathrm{EZ}}$. Figure 14 shows the decrease in $\mathrm{S} / \mathrm{N}$ as the exozodiacal emission increases in the case of a face-on disk; an edge-on disk will increase the surface brightness and resultant noise. As with the interferometer, the effect of zodiacal emission in the target system is to lower the $\mathrm{S} / \mathrm{N}$ by a factor of $2-3$ at $\mu_{\mathrm{EZ}}=10$.

The relative effect of the exozodiacal emission is somewhat more pronounced for the $T P F-C$ than for the TPF-I because the interferometer is dominated by the strong local zodiacal background until very bright exozodiacal levels are observed. The intrinsic background level within the visible-light coronagraph is low (by assumption of an excellent $10^{-10}$ rejection ratio) so that the exozodiacal emission more quickly plays a significant role in setting the system noise.

A more detailed examination of the effects of the exozodiacal emission on the detectability of planets using TPF-C and TPF-I would yield absolute, not relative, sensitivity levels including the effects of disk inclination and confusion by structures, e.g., wakes and gaps, within the zodiacal cloud. These questions lie beyond the scope of this paper.

Angel, J. R. P., \& Woolf, N. J. 1997, ApJ, 475, 373

Aumann, H. H. 1988, AJ, 96, 1415

Aumann, H. H., \& Probst, R. G. 1991, ApJ, 368, 264

Backman, D. E. 1998, in Exozodiacal Dust Workshop, ed. D. E. Backman et al. (Moffett Field: NASA), 107

Backman, D. E., \& Paresce, F. 1993, in Protostars and Planets III, ed. E. H. Levy \& J. I. Lunine (Tucson: Univ. Arizona Press), 1253

Barry, D. C. 1988, ApJ, 334, 436

\section{REFERENCES}

Beichman, C. A. 1998, Proc. SPIE, 3350, 719

Beichman, C. A., \& Velusamy, T. 1999, in ASP Conf. Ser. 194, Working on the Fringe: Optical and IR Interferometry from Ground and Space, ed. S. Unwin \& R. Stachnik (San Francisco: ASP), 408

Beichman, C. A., Woolf, N. J., \& Lindensmith, C. A. 1999, The Terrestrial Planet Finder $(T P F)$ : A NASA Origins Program to Search for Habitable Planets (Washington, DC: NASA)

Beichman, C. A., et al. 2005a, ApJ, 622, 1160 
Beichman, C. A., et al. 2005b, ApJ, 626, 1061 . 2005c, in Protostars and Planets V, ed. B. Reipurth, D. Jewitt, \& K. Keil (Tucson: Univ. Arizona Press), 8574 2006, ApJ, 639, 1166

Bernstein, R. A., Freedman, W. L., \& Madore, B. F. 2002, ApJ, 571, 85 Bracewell, R. N. 1978, Nature, 274, 780

Brott, I., \& Hauschildt, P. H. 2005, in The Three-dimensional Universe with Gaia, ed. C. Turon, K. S. O’Flaherty, \& M. A. C. Perryman (ESA SP-576; Noordwijk: ESA), 565

Brown, R. A. 2005, ApJ, 624, 1010

Bryden, G., et al. 2006, ApJ, 636, 1098

Burns, J. A., Lamy, P. L., \& Soter, S. 1979, Icarus, 40, 1

Butler, R. P., et al. 2004, ApJ, 617, 580

Decin, G., Dominik, C., Malfait, K., Mayor, M., \& Waelkens, C. 2000, A\&A, 357, 533

Dermott, S. F., \& Kehoe, T. J. J. 2004, in ASP Conf. Ser. 324, Debris Disks and the Formation of Planets, ed. L. Caroff et al. (San Francisco: ASP), 143

Dominik, C., \& Decin, G. 2003, ApJ, 598, 626

Draine, B. T., \& Lee, H. M. 1984, ApJ, 285, 89

Eggen, O. J. 1998, AJ, 115, 2397

Fajardo-Acosta, S. B., Beichman, C. A., \& Cutri, R. M. 2000, ApJ, 538, L155

Fischer, D. A., \& Valenti, J. 2005, ApJ, 622, 1102

Garaud, P., \& Lin, D. N. C. 2006, ApJ, in press

Giorgini, J. D. 2005, Asteroid and Comet Field-of-View Search Request

Gonzalez, G. 1997, MNRAS, 285, 403

Gordon, K. D., et al. 2004, Proc. SPIE, 5487, 177 2005, PASP, 117, 503

Habing, H. J., et al. 1996, A\&A, 315, L233 2001, A\&A, 365, 545

Hauschildt, P. H., Allard, F., \& Baron, E. 1999, ApJ, 512, 377

Hayashi, C. 1981, Prog. Theor. Phys. Suppl., 70, 35

Holmes, E. K., Dermott, S. F., \& Gustafson, B. A. S. 2002, in Asteroids, Comets, and Meteors: ACM 2002, ed. B. Warmbein (ESA SP-500: Noordwijk: ESA), 43

Kalas, P., Graham, J. R., \& Clampin, M. 2005, Nature, 435, 1067

Kalas, P., Graham, J. R., Clampin, M. C., \& Fitzgerald, M. P. 2006, ApJ, 637, L57

Kasting, J. F., Whitmire, D. P., \& Reynolds, R. T. 1993, Icarus, 101, 108

Kelsall, T., et al. 1998, ApJ, 508, 44

Kim, J. S., et al. 2005, ApJ, 632, 659

Kurucz, R. L. 2003, in IAU Symp. 210, Modelling of Stellar Atmospheres, ed. N. Piskunov, W. W. Weiss, \& D. F. Gray (San Francisco: ASP), 45

Lachaume, R., Dominik, C., Lanz, T., \& Habing, H. J. 1999, A\&A, 348, 897

Landgraf, M., Liou, J.-C., Zook, H. A., \& Grün, E. 2002, AJ, 123, 2857

Laughlin, G., Bodenheimer, P., \& Adams, F. C. 2004, ApJ, 612, L73

Laureijs, R. J., et al. 2002, A\&A, 387, 285

Lawson, P., Unwin, S., \& Beichman, C. A. 2004, JPL Tech. Rep. 04-014 (Pasadena: JPL)

Lay, O. P. 2004, Appl. Optics, 43, 6100

Lay, O. P., et al. 2005, Proc. SPIE, 5905, 8
Levison, H. F., \& Morbidelli, A. 2003, Nature, 426, 419

Mannings, V., \& Barlow, M. J. 1998, ApJ, 497, 330

Marcy, G. W., Butler, P. R., Fischer, D. A., \& Vogt, S. S. 2004, in ASP Conf. Ser. 321, Extrasolar Planets: Today and Tomorrow, ed. J.-P. Beaulieu, A. L. des Etangs, \& C. Terquem (San Francisco: ASP), 3

Marcy, G. W., Butler, P. R., Frink, S., Fischer, D., Oppenheimer, B., Monet, D. G., Quirrenbach, A., \& Scargle, J. D. 2002, in Science with the Space Interferometry Mission, ed. S. Unwin \& S. Turyshev (Pasadena: JPL), 3

Martin, S. R., Gappinger, R. O., Loya, F. M., Mennesson, B. P., Crawford, S. L., \& Serabyn, E. 2003, Proc. SPIE, 5170, 144

McKee, C. F., \& Taylor, J. H. 2001, S\&T, 101, 38

Meyer, M. R., et al. 2004, ApJS, 154, 422

Montes, D., et al. 2001, MNRAS, 328, 45

Moro-Martín, A., \& Malhotra, R. 2005, ApJ, 633, 1150

Nitschelm, C., et al. 2000, A\&AS, 145, 275

Perrin, M.-N., de Strobel, G. C., Cayrel, R., \& Hejlesen, P. M. 1977, A\&A, 54, 779

Perryman, M. A. C., et al. 1997, The Hipparcos and Tycho Catalogues (ESA SP-1200; Noordwijk: ESA)

Plavchan, P., Jura, M., \& Lipscy, S. J. 2005, ApJ, 631, 1161

Randich, S., Gratton, R., Pallavicini, R., Pasquini, L., \& Carretta, E. 1999, A\&A, 348, 487

Rieke, G. H., et al. 2004, ApJS, 154, 25

. 2005, ApJ, 620, 1010

Rocha-Pinto, H. J., \& Maciel, W. J. 1998, A\&A, 339, 791

Santos, N. C., Israelian, G., \& Mayor, M. 2001, A\&A, 373, 1019

Sasselov, D. D., \& Lecar, M. 2000, ApJ, 528, 995

Shao, M., et al. 2002, in Science with the Space Interferometry Mission, ed. S. Unwin \& S. Turyshev (Pasadena: JPL), 7

Sheret, I., Dent, W. R. F., \& Wyatt, M. C. 2004, MNRAS, 348, 1282

Shevelev, Y. G., \& Marsakov, V. A. 1988, Bull. Cent. Donnees Stellaires, 35, 131

Spangler, C., Sargent, A. I., Silverstone, M. D., Becklin, E. E., \& Zuckerman, B. 2001, ApJ, 555, 932

Stapelfeldt, K. R., et al. 2004, ApJS, 154, 458

Stencel, R. E., \& Backman, D. E. 1991, ApJS, 75, 905

Stern, S. A. 1996, A\&A, 310, 999

Su, K. Y. L., et al. 2005, ApJ, 628, 487 . 2006, ApJ, in press

Traub, W. A., Kasting, J., Shao, M., Johnston, K. J., \& Beichman, C. A. 2006 , in IAU Colloq. 200, Direct Imaging of Exoplanets: Science and Techniques, ed. C. Aime \& F. Vakili (Cambridge: Cambridge Univ. Press), 399

Valenti, J. A., \& Fischer, D. A. 2005, ApJS, 159, 141

Walker, H. J., \& Wolstencroft, R. D. 1988, PASP, 100, 1509

Werner, M. W., et al. 2004, ApJS, 154, 1

Wright, J. T., Marcy, G. W., Butler, R. P., \& Vogt, S. S. 2004, ApJS, 152, 261

Wyatt, M. C., Greaves, J. S., Dent, W. R. F., \& Coulson, I. M. 2005, ApJ, 620, 492 